\title{
Les tombeaux ouverts : montrer les corps saints à la fin du Moyen Âge (1460-1520)
}

Open Graves: Displaying Saints' Bodies at the End of the Middle Ages (1460-1520)

Nicolas Sarzeaud

\section{CpenEdition}

1 Journals

Édition électronique

URL : http://journals.openedition.org/imagesrevues/6911

DOI : 10.4000/imagesrevues.6911

ISSN : 1778-3801

Éditeur :

Centre d'Histoire et Théorie des Arts, Groupe d'Anthropologie Historique de l'Occident Médiéval, Laboratoire d'Anthropologie Sociale, UMR 8210 Anthropologie et Histoire des Mondes Antiques

\section{Référence électronique}

Nicolas Sarzeaud, «Les tombeaux ouverts : montrer les corps saints à la fin du Moyen Âge

(1460-1520) », Images Re-vues [En ligne], 16 | 2019, mis en ligne le 21 décembre 2019, consulté le 03 février 2021. URL : http://journals.openedition.org/imagesrevues/6911 ; DOI : https://doi.org/10.4000/ imagesrevues.6911

Ce document a été généré automatiquement le 3 février 2021

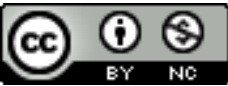

Images Re-vues est mise à disposition selon les termes de la Licence Creative Commons Attribution Pas d'Utilisation Commerciale 4.0 International. 


\title{
Les tombeaux ouverts : montrer les corps saints à la fin du Moyen Âge (1460-1520)
}

Open Graves: Displaying Saints' Bodies at the End of the Middle Ages (1460-1520)

\author{
Nicolas Sarzeaud
}

1 Jacques Lesaige, drapier de Douai, voyageant depuis Rome vers la Terre Sainte, raconte son excursion à Murano :

le 23 mai [1519], nous allâmes nous divertir en la ville de Murano, qui est une petite ville, où il $\mathrm{y}$ a de belles maisons. [...] On y fait tant de verres de cristal que nul ne peut y croire s'il n'y est pas allé. J'en vis faire de toute sorte. Après les avoir vus faire, nous allâmes entendre la messe en l'une des églises [San Pietro Martire]. Quand le prêtre eut dit la messe, on lui apporta des clés. Ensuite il souleva l'autel

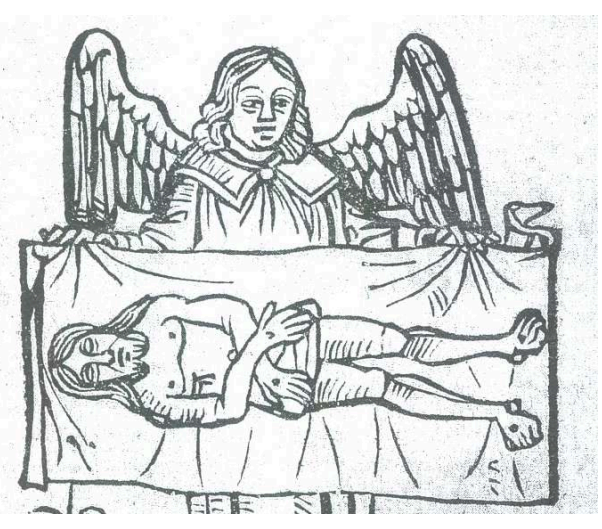
où il avait dit la messe, comme si c'était un coffre, et on nous montra dedans deuxcent-huit corps de petits saints innocents et la plupart avait encore leurs chemisettes : ils étaient entassés par couches dans l'autel et c'est quelque chose de beau. Nous fûmes tous réjouis de voir tant de si beaux jeunes saints. Après, nous revînmes à notre bateau. Et donc nous retournâmes dîner à Venise. Mais soyez sûr que j'avais bon appétit (J.L., p. 45) ${ }^{1}$.

De telles lignes appellent chez le lecteur contemporain des réactions anachroniques, à commencer par un vague dégoût pour la joie que Jacques raconte ressentir à voir des corps d'enfants morts. Nous laisserons ce dégoût ici ; l'expérience sensuelle et dévote de la relique est une relation aux corps des morts bien particulière ${ }^{2}$, dont ce texte nous invite à considérer l'importance, à la fin du Moyen Âge. Dans cette curieuse tombe à couvercle qui s'ouvre et se ferme à volonté3, on doute qu'il ait vu et dénombré jusqu'à deux-cent-huit corps entassés : un autre pèlerin, Jean de Tournai, passé au même 
sanctuaire une trentaine d'années plus tôt, évoque lui cent-cinquante innocents (J.T., f. 98). Combien de nourrissons étaient amassés là, à quoi cela pouvait-il ressembler ? Ici, il est question de "voir ", mais on devine la construction du "voir ", la médiation qui entoure l'acte de montrer.

3 Les pèlerins racontent leurs périples pour aiguiller de futurs candidats au saint voyage, ils décrivent les pays traversés, leurs mœurs et les visites aux reliques des sanctuaires se trouvant sur leurs routes ${ }^{4}$. Ils donnent alors des détails sur la manière dont on les montre. En m'appuyant sur un corpus de récit de pèlerins occidentaux de la fin du Moyen Âge, je propose d'interroger les modulations de ces pratiques de monstration de reliques et leur gestualité. Dans cette enquête, voici nos informateurs, ayant réalisé leurs pèlerinages entre 1460 et $1520^{5}$ et auxquels je renvoie par leurs initiales ${ }^{6}$ : Léon de Rozmital, seigneur Praguois traversant l'Europe entre 1465 et 1467, Hans von Waltheym, patricien d'Halle, en 1474, Felix Fabri, dominicain d'Ulm, en 1480 et 1483, Jérôme Münzer, médecin de Nuremberg, en 1495, Antonio de Beatis, secrétaire du cardinal d'Aragon, le suivant dans son voyage entre 1517 et 1518, Philippe de Vigneulles, bourgeois messin, en 1510, Jacques Lesaige, en 1518-1519 ainsi que le marchand valenciennois Jean de Tournai.

Ce dernier était parti en février 1487, pérégriner vers Rome, Jérusalem et SaintJacques-de-Compostelle où il arrive enfin, en janvier 1488. Là, il se confesse et fait dire une messe conclusive à son impressionnant périple. Une fois célébrée l'eucharistie, il monte par une échelle au saint Jacques sculpté qui surplombe l'autel puis descend dans la confession où le saint est enterré. Il voit le bourdon du saint pèlerin dans une chapelle de la nef, puis une clochette sonne et il emprunte un escalier vers un trésor. «Là, nous fut montré la tête de saint Jacques (J.T., f.271) ${ }^{7}$ ", raconte Jean ; qu'a-til vu, sachant que dès le xvI siècle on met en doute l'existence même de la relique ${ }^{8}$ ? Il ne s'attarde pas à décrire la relique, mais détaille son dispositif :

il y avait dans la chapelle un des officiers de l'église qui avait une robe moitié blanche, moitié vermeille, et il dit en trois langues, à savoir en latin, en allemand et en français, tout haut, que quiconque croyait fermement que le corps de saint Jacques n'était pas enchâssé ou maçonné dans le grand autel de l'église, comme il apparaît aussi sur la lettre ou rouleau que porte l'image sur le grand autel et sur lequel il est dit et écrit en lettres romaines 'Hic jacet [ci-gît] ...', il dit qu'il fait son pèlerinage en vain (J.T., f. 291) ${ }^{9}$.

5 Les spécialistes de l'architecture ont mis en évidence la manière dont l'espace ecclésial distribuait les autels pour faciliter la progression des fidèles d'une relique à une autre ${ }^{10}$. Dans cette progression, une série de gestes rituels concourent à l'accomplissement du pèlerinage au moins autant que le fait de voir les reliques : se déplacer d'un autel à un autre, s'agenouiller et prier, déposer un cierge, un ex-voto, toucher ou faire toucher à son bâton le corps saint. À ces gestes du pèlerin, répondent les gestes et les discours d'officiants aguerris et même polyglottes, capables de guider les nombreux pèlerins venus de tous les pays dans le sanctuaire, d'expliquer ce qui leur est montré, d'encadrer leurs pratiques de dévotion et de les inscrire dans une liturgie, de manipuler les reliquaires et les corps saints; en somme, d'assurer la bonne médiation des reliques.

Ces pratiques de « monstration $»^{11}$ intéressent bien sûr une histoire sociale des reliques, mais aussi une histoire des images. Jean-Claude Schmitt et Jérôme Baschet ont montré que les images médiévales étaient des images-corps et des images-objets, indissolublement ${ }^{12}$. Le constat appelle sa réciproque, tout particulièrement pour les corps saints, os, fragments d'os, textiles ou autres objets de la vie du saint; les riches 
reliquaires dans lesquels ils sont insérés en font des images ostentatoires et certains de ces reliquaires donnent à voir leur contenu par des monstrances, insistant sur leur visualité, comme l'a montré Pierre-Olivier Dittmar ${ }^{13}$. Les pratiques de monstrations, dans leur spectre large, manifestent elles aussi la pleine nature d'images de ces corps saints. Dès lors, on trouve parmi la foule des spectateurs auxquels on montre les reliques un poste d'observation pour une histoire du voir et comme l'écrit Philippe Cordez, une histoire de l'art ${ }^{14}$.

\section{Désir de voir et pratiques d'ostension}

7 Les récits de pèlerinages sont précieux dans cette perspective, car le droit canon et les grands traités liturgiques sont peu bavards sur la monstration des reliques. Le concile de Latran IV de 1215 évoque bien pour les interdire les «ostensions à but lucratif (ostensiones venales) » d'anciennes reliques hors de leurs châsses, cependant il n'est sans doute pas question ici des liturgies d'ostension, mais plutôt des expositions de reliques dans le but de les vendre ${ }^{15}$.

Ce silence s'explique peut-être par la singularité de ce rituel dans le répertoire liturgique; la consécration et l'élévation des saintes espèces se font dos aux fidèles, tandis qu'en montrant le corps saint le clergé le tourne vers les laïcs, du chœur vers la nef, voire même de l'église vers la ville. Pour autant, on ferait fausse route en associant ces pratiques à une religion populaire ou sécularisée : le pape procède lui-même à des ostensions de la sainte Face du Christ ou des chefs de Pierre et Paul à Rome, notamment à l'occasion des jubilés, à partir de 1300 (fig. 1$)^{16}$. Les chroniqueurs, mais encore les archives des chapitres et des villes documentent dans certains sanctuaires de fastueuses ostensions se multipliant au cours des $\mathrm{XIII}^{\mathrm{e}}$ et $\mathrm{XIV}^{\mathrm{e}}$ siècles, comme celles étudiées par Hartmut Kühne dans l'Allemagne de la fin du Moyen Âge ${ }^{17}$. 


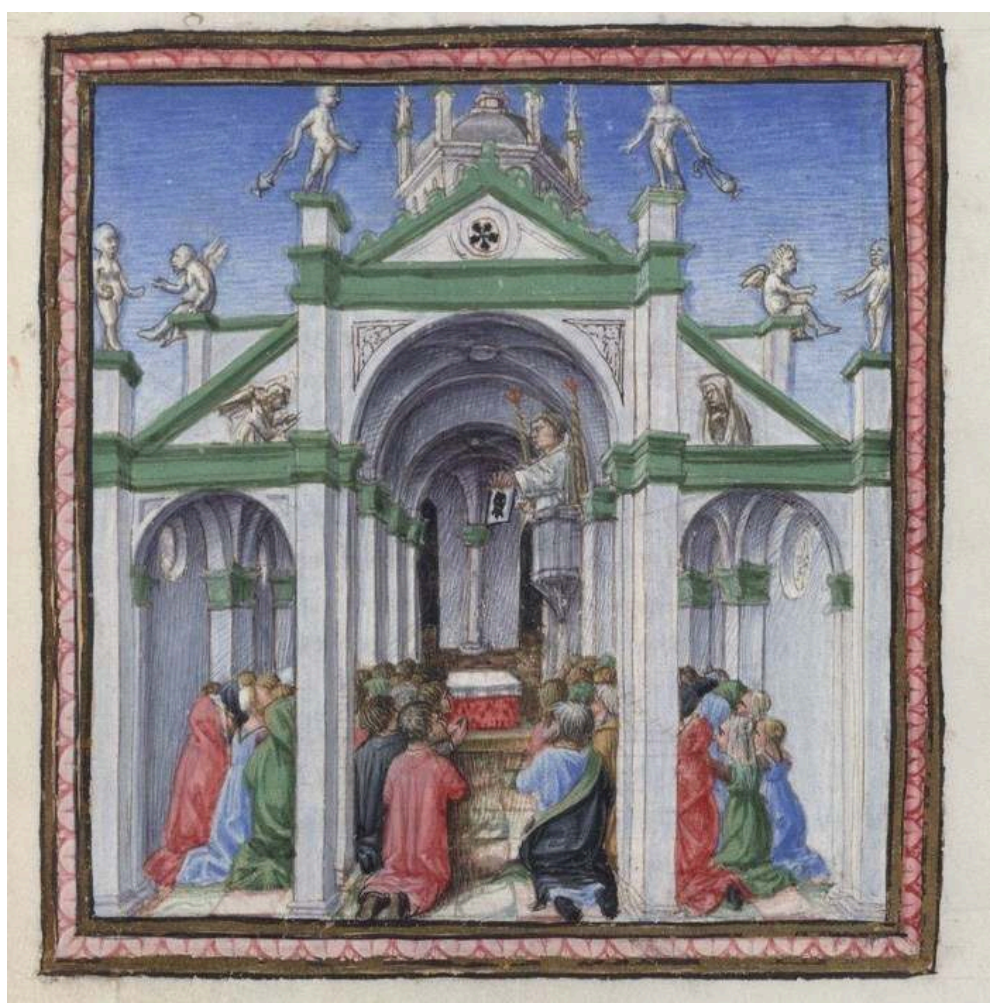

«Le pape Sixte IV montrant la Véronique en 1475 », Fasti Christianae Regionis de Ludovico Lazzarelli, fin du XVe siècle, New Haven, Yale Univesity, Beinecke Rare Book and Manuscript Library, ms.391, $f^{\circ} 41$.

D’après la base de données https://veronicaroute.com.

De quand peut-on dater l'émergence de ces liturgies? Le parallèle entre l'affirmation du rituel d'élévation de l'hostie au début du XIII ${ }^{\text {e }}$ siècle et la multiplication des cérémonies d'ostensions de reliques ${ }^{18}$ est sans doute un peu rapide; des reliquaires à monstrances existent aux siècles précédents ${ }^{19}$ et la première mention d'une cérémonie $\mathrm{d}^{\prime}$ ostensio date du XI $\mathrm{XI}^{\mathrm{e}}$ siècle, pour le saint Suaire de Compiègne ${ }^{20}$. Dès les $\mathrm{VIII}^{\mathrm{e}}-\mathrm{IX}^{\mathrm{e}}$ siècles un calendrier des reliques locales se met en place célébrant la depositio, la translatio ou l'elevatio de la relique ${ }^{21}$, autant de rituels dans lesquels la visibilité des reliquaires et des reliques jouait un rôle.

De la sorte, si on cherche à remonter l'histoire chrétienne pour identifier les prémices d'une visualité des reliques, on est tiré toujours plus amont jusqu'à l'apparition même du culte des reliques : ne les trouve-t-on pas à l'époque carolingienne, où se développe une architecture au service des reliques et la « liturgie du spectacle » décrite par Carol Heitz $^{22}$ ? Ne doit-on pas remonter à l'antiquité tardive avec Georgia Frank, qui met en évidence l'importance du visuel dans l'expérience des pèlerins dès les $\mathrm{IV}^{\mathrm{e}}-\mathrm{VI}^{\mathrm{e}}$ siècles ${ }^{23}$ ?

Une autre problématique d'historicisation émerge alors, perpendiculaire à la première : que signifie voir pour un fidèle de chacune de ces époques? Ici, nous prenons le parti de laisser de côté ce débat pour interroger le montrer, les processus et les pratiques donnant les reliques à voir aux pèlerins. Nous nous intéresserons donc aussi bien aux monstrations informelles destinées à un petit groupe de pèlerins visitant le sanctuaire qu'au faste des grandes cérémonies d'ostensions publiques, pour comprendre comment on accède aux reliques, avec quels ornements et par quels gestes on les montre, 
comment ceux qui y assistent participent à ces épiphanies et comment l'iconographie se saisit des monstrations de reliques.

\section{Puis-je voir vos reliques?}

12 Hans von Waltheym, traversant la Provence, souhaite voir le corps de sainte Anne, à Apt :

alors que nous nous étions défaits à l'auberge à Apt, nous allâmes à l'église. Là, on m'apprit que je devais demander le doyen du chapitre. Ce que je fis ensuite. Il vint me trouver à l'église. C'est un chartreux qui a été longtemps à Rome. C'était un homme raisonnable, honnête, avec lequel je parlai en latin et je lui dis que j'étais venu de pays lointain pour la très sainte Anne. Je lui demandai donc de bien vouloir m'apporter son aide car j'aimerais voir la sainte relique, etc. Aussitôt il envoya chercher en ville quatre chanoines et quatre magistrats de la ville qui avaient les clés et nous conduisit dans une grande crypte. Là ils ouvrirent toutes les serrures et nous montrèrent la tête de sainte Anne et sa sainte dépouille et encore beaucoup d'autres objets sacrés (H.W., p. 539).

13 Si on comprend la monstration comme le processus qui satisfait le souhait du fidèle d'accéder à la relique, on doit intégrer ce premier volet de médiation, informel : pour voir une relique, il faut déjà savoir où se trouve le sanctuaire ${ }^{24}$, à quel moment elle se montre ou à qui s'adresser pour obtenir une monstration. Les hôtes des pèlerins, comme ceux de Jean de Tournai à Saint-Sernin de Toulouse ${ }^{25}$, mais aussi les guides ou les "patrons", payés par les pèlerins pour organiser le saint voyage, jouent ce rôle d'informateurs voire d'entremetteurs ${ }^{26}$.

Pour répondre à ces demandes, les sanctuaires mettent en place différentes stratégies organisant l'accès aux reliques. Ainsi, Philippe de Vigneulles décrit le système des ostensions rhénanes, qui ont lieu tous les sept ans à Aix-la-Chapelle. Aucune monstration des principales reliques de la cathédrale n'est possible hors de ces dates ; les magistrats s'en excusent auprès de la délégation bohémienne de Léon de Rozmital (L.R., p. 152). De même, Philippe revenant à Metz par Trêves raconte y avoir visité presque toutes les églises de la ville : « mais on ne nous montrait aucune relique, car ils les montrent en d'autres temps et saisons ${ }^{27}$ (P.V., p. 184).»

Inversement, dans certains sanctuaires particulièrement courus, les reliques sont accessibles très régulièrement aux fidèles; non seulement le fastueux reliquaire des Rois Mages de Cologne est visible dans la cathédrale et une grille permet de voir son contenu $^{28}$, mais les reliques font l'objet d'une monstration quotidienne (J.M.', p. 336), système qu'on retrouve notamment pour une Vierge peinte par saint Luc à Notre-Dame de Lorette (J.L., p. 33).

À Apt, Hans décrit une monstration accordée au cas par cas, nécessitant une concertation avec les magistrats de la Ville qui détiennent une partie des clés. Jean de Tournai note cette pratique de partage des clés entre la Ville et le sanctuaire comme une particularité provençale ${ }^{29}:$ «En tout le pays de Provence, on ne peut voir aucun sanctuaire sans avoir avec les trésoriers des églises un homme de la justice des villes, car, comme on vous le dirait, les prévôts ou échevins des villes ont une clé des trésors (J.T., f. 276) ${ }^{30}$. » Les charges des églises médiévales ont des contours mouvants, ici Jean attribue au trésorier cette fonction de faire visiter les reliques aux pèlerins ${ }^{31}$. Ce n'est pas un mince enjeu: plus loin, Jean se plaint de la coutume "fort mauvaise et 
dangereuse ", pratiquée par certains sanctuaires provençaux, de réclamer une somme d'argent aux fidèles qui veulent visiter leurs reliques ${ }^{32}$.

17 À Saint-Maximin, Hans von Waltheym souhaite voir le crâne de Marie-Madeleine. Il s'adresse à un prieur strasbourgeois qui le guide jusqu'à la crypte de la basilique où il sort de sa loge le masque-reliquaire ${ }^{33}$. Le prieur ne peut pas l'ouvrir, car les magistrats, absents, en ont les clés : par chance, le dimanche suivant se déroule une ostension publique du reliquaire, masque ouvert, laissant voir le visage de la sainte à travers un cristal. Le jour de l'ostension, après les vêpres, le prieur lui montre une dernière fois la relique (H.W., p. 500-507).

Dans notre corpus, trois autres chroniqueurs décrivent leurs tête-à-tête avec MarieMadeleine, avec des modalités presque chaque fois différentes. On leur montre le reliquaire: ouvert sur l'autel principal de l'église haute et en présence de tous les fidèles (H.W., p. 502-507) ; ouvert dans la première crypte, avec un dispositif liturgique (J.T., f. 273-274) ; fermé dans la seconde crypte, sans liturgie (H.W., p. 500-501 et 506); ouvert dans la seconde crypte (J.M., p. 588-589, A.B., p. 247).

Dès lors, une typologie simple des politiques d'accès aux reliques serait inopérante, en raison de la diversité des pratiques, offrant des modulations fines d'un sanctuaire à un autre et au sein d'un même sanctuaire. Plus précisément, c'est une dichotomie entre des reliques accessibles et inaccessibles que déjouent ces exemples. C'est le paradoxe de la crypte, soulevé par Christian Sapin ; étymologiquement liées au secret, les cryptes sont pourtant dès l'époque romane des lieux d'assemblées et de circulation fréquente, comme on le voit à Saint-Maximin ${ }^{34}$. Sur ce mode, l'accès à des lieux et des reliques réputés « inaccessibles » est sans cesse négocié et les épais verrous souvent levés.

On peut prendre le cas classique de la Sainte-Chapelle de Paris, longtemps qualifiée de chapelle "privée "; dans notre corpus, Jérôme Munzer est guidé par les chanoines jusqu'aux reliquaires de la Passion, dans l'église haute. Ils s'excusent de ne pas pouvoir les ouvrir en raison de l'absence du roi et du régent qui en détiennent les clés (J.M.', p. 290-291). Comme l'a bien montré Meredith Cohen, cette chapelle n'était certainement pas une chapelle " privée » fréquentée exclusivement par le roi et ses proches ${ }^{35}$.

\section{Les retentissantes ostensions secrètes}

Dans notre documentation, on retrouve régulièrement un système dual de monstrations, avec d'une part les ostensions publiques, limitées à des dates fixes, et des monstrations exceptionnelles accordées à des fidèles désirant voir les reliques. On trouve ce système réglementé pour le saint Suaire de Toulouse à la fin du XIV siècle ou pour celui de Besançon au XVI ${ }^{\mathrm{e}}$ siècle, réservant aux personnages les plus importants ou venant de loin ces ostensions qualifiées de secrete $e^{36}$; sans doute ne faut-il pas entendre ostensions "secrètes ", au sens de "cachées ", mais plutôt de " distinctes ", "à part ". La fréquence avec laquelle on déroge à la règle, le large éventail de raisons pour lesquelles on le fait et la nature en partie publique de ces ostensiones secretes empêche là encore de les qualifier de « privées ».

Les dérogations sont accordées aux personnages de haut rang; le cardinal d'Aragon se voit ouvrir des portes restées closes aux pèlerins plus modestes, celles de la SainteChapelle de Chambéry par exemple (A.B., p. 208-210, au contraire de J.L., p. 8). Toutefois, nos autres témoins, clercs ou bourgeois lettrés, obtiennent aussi de multiples 
monstrations exceptionnelles, le statut de pèlerin étant dérogatoire ; ainsi Jacques Lesaige, déçu que le corps de saint Roch à Venise ne lui ait pas été montré lors de l'office dominical, s'entend répondre qu'il lui faut revenir quand il le souhaite et qu'en tant que pèlerin du saint voyage, la relique lui sera montrée (J.L., p. 44-45).

Ainsi, les pèlerins obtiennent régulièrement des monstrations qui leur sont réservées, mais qui loin d'être «secrètes » réunissent d'importants groupes de voyageurs (J.L., p. 160-161). Jean de Tournai assiste à ce type de cérémonie, à Rome :

le pape nous fit montrer la sainte Véronique et tous les reliquaires de Rome comme on a coutume de les voir pendant la Semaine sainte, mais ce fût à huis clos, c'est-àdire que lorsque nous, les pèlerins, étions entrés dans les églises, on fermait les huis (J.T., f. 263) ${ }^{37}$.

Ce privilège accordé aux seuls pèlerins, excluant les paroissiens, peut générer quelques tensions, que Jean décrit à Zadar pour le corps de saint Siméon :

aussitôt que ceux de la ville virent que les prêtres allaient le montrer, aussitôt le peuple de la vallée vint à si grande force que nous ne pûmes le voir.

Les clercs sévissent alors :

ils prirent de longs fouets et frappèrent à tort et à travers, aussi bien sur leurs visages que sur leurs têtes, et encore ils ne firent cas de rien, car il nous manquait là un très grand espace. Et donc voyant cela ils les expulsèrent jusqu'à la porte donnant sur la rue et la fermèrent avec force. Et après ils vinrent à la porte donnant dans l'église et expulsèrent ceux qui étaient dans la dite chapelle en dehors avec force (J.T., f. 107-108) ${ }^{38}$.

Les paroissiens n'ont-ils aucun moyen d'obtenir des ostensions exceptionnelles ? Par sa nature, notre corpus ne fait pas état de ce type de dérogations, mais elles existent ; en 1544, le chapitre de Besançon délibère sur la demande d'un des gouverneurs de la ville d'amener le saint Suaire jusque chez lui, auprès de sa femme, malade. On refuse et on décide que ça ne se fera plus $^{39}$; dans des circonstances exceptionnelles comme la maladie d'un paroissien, des pratiques de monstration sortant du cadre ordinaire ont pu se pratiquer.

On distingue ainsi un nuancier de "régimes de monstrabilité » de la relique. Si on s'attachait à en faire une typologie, ce serait sous la forme d'un tableau à multiples entrées; à qui montre-t-on la relique ? À quel moment de la journée, du calendrier ? Dans quel lieu (la crypte, une chapelle, la nef, la place de l'église, la maison d'un paroissien) ? Quelles expériences le fidèle peut-il faire de la relique (voir une image, voir le reliquaire, voir la relique nue par une grille ou une monstrance, voir la relique hors de son reliquaire, toucher la relique, etc.)? Quel dispositif accompagne la monstration (y a-t-il une messe ou pas, des chants, des luminaires, etc.) ? Une dernière distinction nous semble particulièrement importante ; est-ce la relique qui sort de son lieu pour entrer dans l'espace des fidèles, la nef ou la place de l'église, ou est-ce le fidèle qui est autorisé par le clergé à pénétrer les espaces de la relique?

\section{Franchir les seuils}

Hans von Waltheym décrit sa visite à Saint-Antoine-l'Abbaye :

dans la cathédrale se trouve la sainte dépouille du fidèle et grand sauveur saint Antoine, au-dessus du maître-autel dans un cercueil décoré d'argent, d'or, de pierres précieuses, et le cercueil se trouve sur quatre hautes colonnes en pierre, si haut que l'on voit le cercueil dans l'église et la cathédrale, au-dessus du retable du 
maître-autel [...]. Idem, à Saint-Antoine on montre le bras de saint Antoine : il est travaillé dans de l'or et des pierres précieuses et l'os du bras est nu, de sorte qu'on peut l'embrasser dessus et sur le côté. C'est le matin et le soir qu'on le montre, et on le met sur un autel. Dans l'abside il y a un prêtre auprès du bras (H.W., p. 466). médiévale, limitant l'accès des laïcs au chœur, aux chapelles et aux cryptes. Pour voir la plupart des reliques, la médiation d'un clerc est donc nécessaire, même s'il ne fait qu'ouvrir les portes d'une chapelle, autoriser les pèlerins à y entrer et les surveiller ${ }^{40}$. Ici, le tombeau surélevé visible depuis la nef permet une première expérience du corps de saint Antoine, le clergé contrôlant un second niveau d'intimité, plus recherché ; voir de près et toucher l'os de son bras.

Ce jeu de partitions s'appuie sur le système d'analogies efficaces qui fonde le culte médiéval des reliques, à l'œuvre dans notre corpus. Non seulement la dépouille d'un saint y apparaît comme le corps agissant du saint, mais un fragment de sa dépouille est désigné comme le corps du saint, si bien qu'un saint dont le corps a été loti agit à divers degrés dans plusieurs corps disséminés, ici au sein d'un même sanctuaire.

Dans l'église haute de Saint-Nicolas de Bari, Jean de Tournai décrit une toile peinte figurant le saint : elle « fut amenée sur le char avec le corps saint [...].» Or, « quand on ne peut descendre en bas [dans la crypte] pour saluer le saint, on allume [des luminaires] et on implore le saint devant cette peinture (J.T., f. 254) ${ }^{41}$. » Dans l'espace $\mathrm{du}$ sanctuaire un feuilletage des expériences de la relique s'organise, s'appuyant sur la partition de la dépouille du saint ainsi que sur des images de la relique ${ }^{42}$.

Si on obtient de descendre saluer saint Nicolas, on emprunte un large escalier de vingt et un degrés menant à une porte close. Une fois déverrouillée on accède, cinq marches plus bas, au chœur dans lequel le saint repose. Là se trouve un pilier ayant contenu le corps du saint : «Ce pilier est enclos de fer et il y a un verrou pour entrer dedans. Et il est dit qu'une personne qui serait en état de péché mortel ne pourrait pas y entrer, mais je n'y entrai pas (J.T., f. 254) ${ }^{43}$. » Jean de Tournai a le droit de visiter la crypte, mais n'entre pas dans le pilier.

Une dernière strate autour de la relique lui est accessible, deux battants d'argent sur l'autel s'ouvrent et donnent sur un nouveau seuil :

on ouvre encore par terre comme une grande salière dont le couvercle est en laiton dans lequel il y a un crucifix, et par un trou ou passage, qui est environ aussi grand que ma paume, je regardais comme pour chercher à voir le corps du glorieux saint Nicolas, mais on n'y perçoit rien, sinon quand on a une chandelle, alors on voit tout en bas, ou avec une lampe, rendant une grande clarté, qui est pleine d'huile (f. 255r).»

Si Jean ne peut pas toucher directement le corps, l'huile d'une lampe descendue auprès de la relique joue ce rôle de médiatrice :

on en donne à chaque pèlerin, une ampoulette et pour ma part je trouvai la manière d'en avoir trois (ibidem $)^{44}$.

La mise en visibilité de la relique n'est qu'un moment, ici incertain, d'une progression ritualisée dans l'épaisseur du sanctuaire. Le fidèle, guidé par le médiateur et son trousseau de clés, qu'on imagine épais, franchit plusieurs seuils auxquels peuvent répondre chaque fois des stations réglées par un rituel : célébrer une messe basse sur un autel intermédiaire, déposer une offrande, toucher ou faire toucher une image ou un reliquaire secondaire. À Compostelle par exemple, le pèlerin ayant communié touche la statue de saint Jacques au-dessus de l'autel, avant de poursuivre la progression décrite 
par Jean de Tournai, passant par le corps, le bourdon et la tête du saint (J.T., f. 281). Les nombreux pèlerins se suivant à chacune de ces étapes accomplissent leur pèlerinage par une séquence de gestes qui, sans être sacramentels, présentent bien une «efficacité symbolique ", selon le lexique de Claude Lévi-Strauss utilisé par Jean-Claude Schmitt ${ }^{45}$.

À Bari, cette visite gestualisée du fidèle, franchissant un seuil après l'autre jusqu'au corps saint, structure l'espace comme une poupée gigogne ou comme un oignon, pour reprendre la métaphore d'Alfred Gell ${ }^{46}$, le fidèle cherchant toujours à atteindre la couche suivante. Cette stratification de l'espace de la relique permet une hiérarchisation de l'accès au corps saint, matérialisant une stratigraphie et une hiérarchie sociale. À Saint-Maximin, un panneau interdit aux femmes d'entrer dans les cryptes contenant le corps de Madeleine et de ses compagnes (H.W., p. 500); leurs visites s'arrêtent à l'autel de saint Maximin. À Venise, le matin de l'Ascension, Jean de Tournai assiste au défilé spectaculaire de nobles femmes vénitiennes se pressant dans une chapelle contenant un crucifix miraculeux, l'un des trois jours de l'année où elles sont autorisées à y pénétrer (J.T., f. 82 ${ }^{47}$.

\section{L'ornement en mouvement}

Le 13 juillet 1510, à Metz, quinze cavaliers prennent la route de Luxembourg avec Philippe de Vigneulles, pour aller « au grand pardon Notre Dame à Aix en Allemagne, qui se tient tous les sept ans (P.V., p. 173) ». Durant ces festivités sont montrées quatre reliques textiles de la cathédrale d'Aix-la-Chapelle ; la tunique de la Vierge, les langes et le suaire du Christ, le linge ayant recueilli la tête de Jean-Baptiste. Au même moment, on expose aussi saint Servais à Maastricht, saint Corneille à Kornelimünster, sainte Anne à Düren. Philippe visite encore les Rois Mages et les Ursulines de Cologne avant de rentre par Trêves, soit un parcours d'environ 540 kilomètres (fig. 2). Il arrive à Aix-laChapelle la veille au soir de l'ostension :

[...] nous trouvâmes tant de monde sur le chemin que c'était chose merveilleuse. Quand nous vînmes sur la montagne au-dessus d'Aix, nous vîmes qu'il semblait que toute l'église était en feu et en flammes, par la force des lampes qui brûlaient autour de l'église et c'était presque la nuit de sorte que les lampes se voyaient mieux, et toutes les grosses cloches sonnaient ce qui était une belle chose à voir et à entendre du dessus de la montagne (p. 174) ${ }^{48}$. 
Figure 2

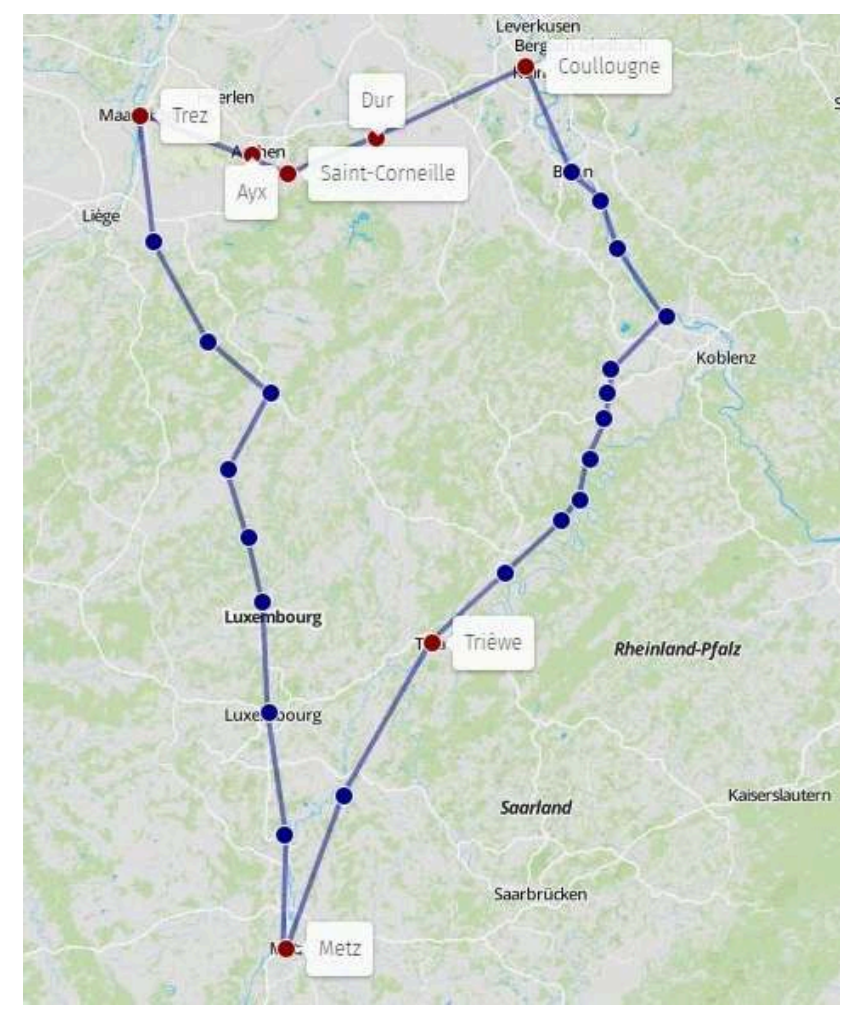

«La saison des reliques », carte du parcours de Philippe de Vigneulles lors des ostensions rhénanes. Openstreetmap - Nicolas Sarzeaud http://umap. openstreetmap. fr/ca/map/la-saison-des-reliques-levoyage-de-jacques-de-vig_305546\#8/50.066/6.422

La ville est comble : Philippe décrit la difficulté à trouver des lits disponibles dans les auberges, "l'horrible presse " dans l'église, où on n'a pas la place pour se mettre à genoux, les clercs qui utilisent des perches pour récupérer les offrandes, les compagnies de pèlerins qui se tiennent par un pan de robe et suivent l'un des leurs bourdon levé afin de rester groupés dans la marée humaine. Le groupe messin paye pour prendre place sur le balcon d'un habitant : «nous avions la vue sur la place d'un des côtés de l'église, de là nous voyions un si grand peuple sur la dite place qu'on ne voyait que des têtes et encore autant sur les maisons (P.V., p. 174-175 et 177) ${ }^{49}$." L'église brille de feux de joie, la place est noire de monde, la musique tonne; on va montrer les reliques.

Dans «L'ornement est-il animiste? ?, Thomas Golsenne théorise le couple formé par l'objet puissant et son ornement :

L'ornementalisation d'une forme ou d'un objet permet de leur attribuer une certaine puissance. L'ornement offre la possibilité d'animer des objets inanimés, même si ses effets sont souvent attribués, dans des cas concrets et des situations culturelles spécifiques, à une cause transcendante ou à l'objet orné. L'art chrétien médiéval, fondé en grande partie sur l'opposition entre la figure humaine et l'ornement divin, en fournit de nombreux exemples. Ce processus, Gell, [dans Art et Agency ${ }^{50}$, le nomme attribution d'agency, qu'on peut traduire ici par " animation ", c'est-à-dire que l'objet ainsi animé par l'ornement se voit considéré comme un acteur vivant, entretenant des relations sociales avec son environnement. Un objetacteur qui, grâce à l'ornement, attire à lui les regards, rayonne vers les autres : sa puissance est son champ d'attraction, l'étendue de son aura ${ }^{51}$. 
Les reliques sont pourvues d'une puissante agency ; à travers elles, on met le fidèle en la présence agissante de la personne sainte et de son pouvoir miraculeux, sa virtus. La ville en fête, la presse des fidèles, manifestent déjà ce champ d'attraction: un dispositif ornemental d'un faste correspondant doit l'accompagner pendant l'ostension. Après un sermon et une confession générale prononcée par un clerc depuis la galerie extérieure de la cathédrale, le rituel commence :

On voit venir beaucoup de torches et de flambeaux allumés et puis viennent beaucoup de gens d'Église, tous revêtus de riches habits avec de riches croix et eau bénite et riches encensoirs d'or et d'argent, et en belle ordonnance ils viennent le long des allées [la galerie extérieure de l'église] et au milieu d'eux, du luminaire et des encensoirs, il y a deux prélats bien vêtus d'or et d'argent qui portent sur leurs épaules un long bâton rond, comme une lance, doré d'or fin, sur ce bâton est mise et posée la précieuse et digne chemise de Notre Dame et elle est pliée en plusieurs plis sur leur bâton, et ils le portent comme on porterait un reliquaire, et sur la chemise il y a un beau drap de soie et au-dessus de ce drap de soie un beau drap d'or, et ils vont ainsi cheminant en belle ordonnance jusqu'à l'un des lieux décidés, là où le sermon a été fait; et là donc ils s'arrêtent, avec une grande révérence, découvre la chemise du drap d'or et de celui de soie et donc tout le peuple est à genoux, tête découverte et mains jointes. [...]

Et donc les prélats prennent la chemise qui est ainsi pliée et en grand honneur et révérence ils la laissent aller de ses plis et l'étendent de tout son long au dehors des allées sur un autre drap d'or, à la vue de chacun, et alors on dirait que tout le monde tremble en raison du grand bruit des cornets et du cri des hommes et femmes qui crient miséricorde, et il n'y a pas d'homme dont les cheveux ne se dressent pas sur la tête et dont les larmes ne viennent pas aux yeux. (P.V., p. $175-176)^{52}$.

41 On retrouve plusieurs éléments décrits ici dans d'autres ostensions de notre corpus, par exemple l'ostension du chef de Marie-Madeleine dans la crypte de Saint-Maximin: "quand on doit le montrer, on va d'abord sonner une clochette et ensuite viennent en premiers les novices et les trésoriers de l'église, tous revêtus, avec tous des torches, des chandelles allumées et de l'encens, fort honorablement, et là ils chantent l'antienne 'Maria ergo...53', puis font la collecte (J.T., f. 273) ${ }^{54}$ ». Ainsi on retrouve régulièrement des luminaires, des cloches et clochettes ou encore des offrandes (dons, ex-voto).

Parmi ces éléments, il faut naturellement insister sur le reliquaire. Pour cette enquête nous utilisons les descriptions de monstration riches de détails, mais le plus fréquemment, les pèlerins se contentent d'une phrase témoignant que le corps de tel ou tel saint leur a été montré, faisant une double assimilation : du fragment au corps entier, mais aussi de la relique à son reliquaire. En effet, nos pèlerins ne font le plus souvent aucune différence entre la monstration du reliquaire et la monstration de la relique hors de son reliquaire ; comme l'écrit Jean-Claude Schmitt, « le reliquaire fait la relique $^{55}$ ». Mieux, il arrive que les pèlerins s'appesantissent sur la description d'un reliquaire sans préciser l'identité du saint qu'il accueille (par exemple A.B., p. 52-53). Plus qu'une métonymie, il y a bien une complète analogie entre la relique et son reliquaire.

Chaque élément de ce dispositif a reçu ${ }^{56}$ ou mériterait de recevoir une étude monographique au titre de sa participation à l'ornement des corps et images saintes médiévales; on peut citer dans notre corpus les textes (authentiques de reliques, liste des reliquaires, textes de prières), les images ${ }^{57}$, les défenses (verrous, barres, gardes) qui protègent la relique ou l'architecture dans laquelle la relique apparaît : chacun de ces éléments accuse et manifeste l'importance et le pouvoir de la relique. 

Philippe de Vigneulles insiste sur la «belle ordonnance (p. 175-176)» du clergé qui accompagne la relique : le bel agencement du corps des clercs les uns par rapport aux autres, par rapport à la relique et la progression harmonieuse de cet ensemble.

Nos pèlerins décrivent rarement des processions, liturgie qui s'adresse plutôt à la communauté locale, mais on doit noter les ressemblances entre ces deux rituels activant la relique. Pour ce qui nous occupe, certaines monstrations présentent un moment processionnel, dans lequel le clergé porte la relique et son dispositif jusqu'au lieu où on les montre. À l'inverse, la procession comporte aussi une exposition de la relique dans les différents lieux où elle est transportée. Dans le cadre de ces deux rituels, le reliquaire se trouve activé par sa visibilité temporaire et animé par le mouvement des processionnaires. 
Comme pour la procession ${ }^{59}$, il faut souligner la dimension sociale du ritue d'ostension qui donne à voir autour de la relique les clercs en habits, les grands laïcs, les hommes en armes et les fidèles; on retrouve tout ce monde chrétien représenté par rapport à la relique dans un livret de pèlerinage nurembergeois (fig. 3).

Figure 3

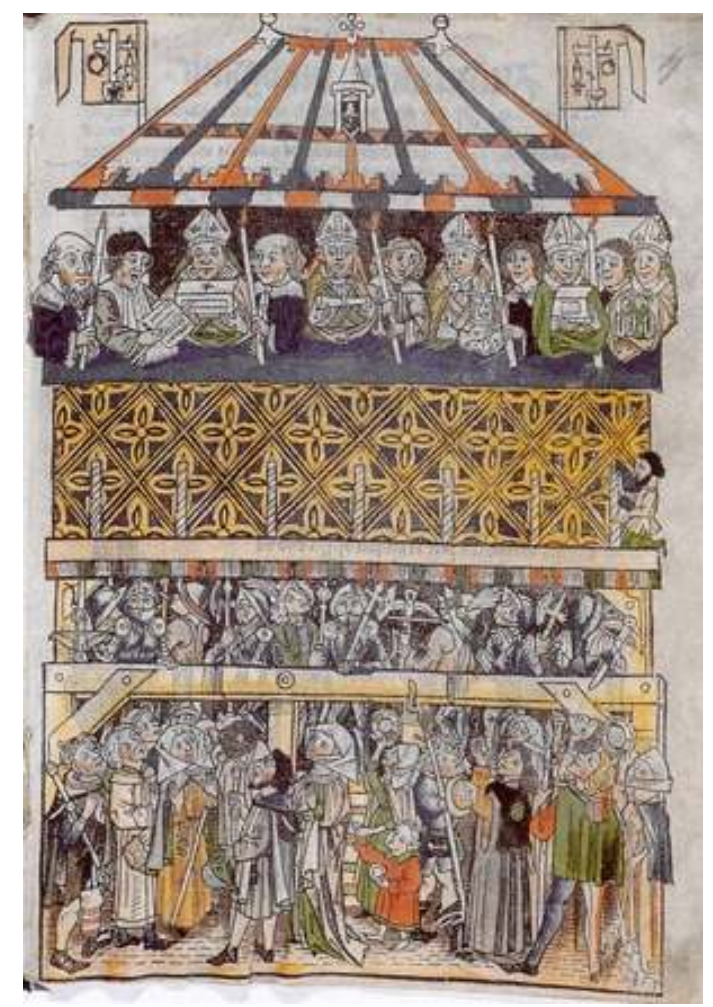

Ostension des reliques de Nuremberg à la Maison Schopperschen, 1487, gravure sur bois, Staatsarchiv de Nuremberg, Rst. Nürnberg Handschriften 399a.

Wikimedia Commons.

Pierre-Olivier Dittmar parle d'un "pacte de visibilité » entre la relique exposée et la communautét ${ }^{6}$. Si la relique est un objet-personne animé, on doit considérer son point de vue; il convient que la communauté se présente devant elle dans sa plus belle ordonnance. Cet ordre idéal, c'est là encore une stratification organisant la société entre ses plus éminents représentants, qui prennent place du côté de la relique et auprès d'elle, et un peuple en révérence. Mais ce peuple lui-même est un peuple idéal, expurgé : ainsi, les sindics de Chambéry ordonnent en 1514 aux « coquins et bélîtres ", mais à tous ceux qui n'ont pas de métiers, de quitter la ville le jour de l'ostension ${ }^{61}$.

Une fois la relique portée jusqu'au lieu où elle est montrée, on ouvre les reliquaires et lève les tissus qui enveloppent la relique. Valeria Genovese a finement étudié les nombreux voiles qui séparent l'espace des laïcs et l'espace des clercs, couvrent les images, les reliquaires et les reliques ${ }^{62}$. Dès lors, le geste d'ouvrir les tentures et de dévoiler les objets prend une grande puissance sémantique, associé au déchirement $\mathrm{du}$ voile du temple attendu pour la fin des temps. Ainsi dans le Christ de douleurs de Meister Francke, des anges font tomber de précieux textiles devant et derrière le corps blessé du Christ, signifiant son avènement (fig. 4). Faire tomber le textile qui entoure une 
relique, c'est au sens propre et figuré une revelatio ${ }^{63}$; c'est donner à voir l'envers sacré du monde.

\section{Figure 4}

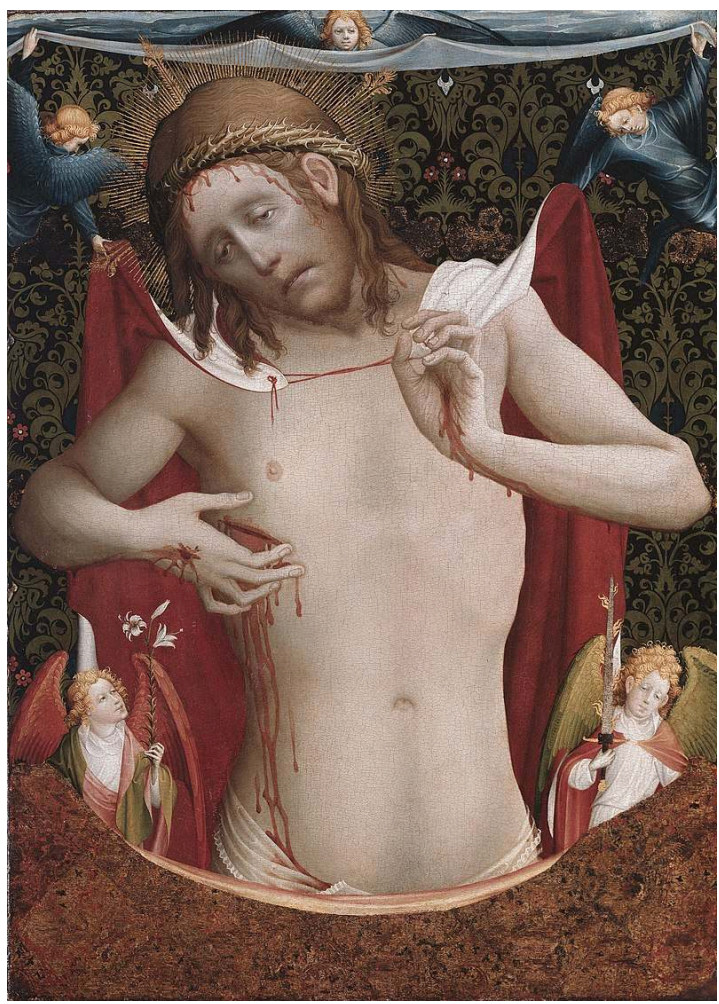

Maître Francke, Christ souffrant, c.1430, Hamburger Kunsthalle.

Wikimedia Commons.

Dans l'iconographie, les reliques sont généralement manipulées dans un linge qui couvre la partie inférieure, ainsi dans les enluminures figurant la sainte Croix portée par saint Louis et le vase du saint Sang de Westimnster porté par Henri III dans les enluminures de la Chronique de Mathieu Paris ${ }^{64}$ (fig. 5). Cette règle de ne découvrir l'objet qu'en partie est connue pour d'autres accessoires liturgiques ${ }^{65}$. À tout le moins, des gants viennent prendre le relais de l'enveloppe textile (on les voit par exemple dans l'ostension nurembergeoise, fig. 3). Ce dispositif textile manifeste la "surmatérialité " de la relique, au sens de Caroline Bynum ${ }^{66}$, jouant ici un rôle de couche protectrice : la relique ne peut se présenter au monde que protégée de son impureté. 


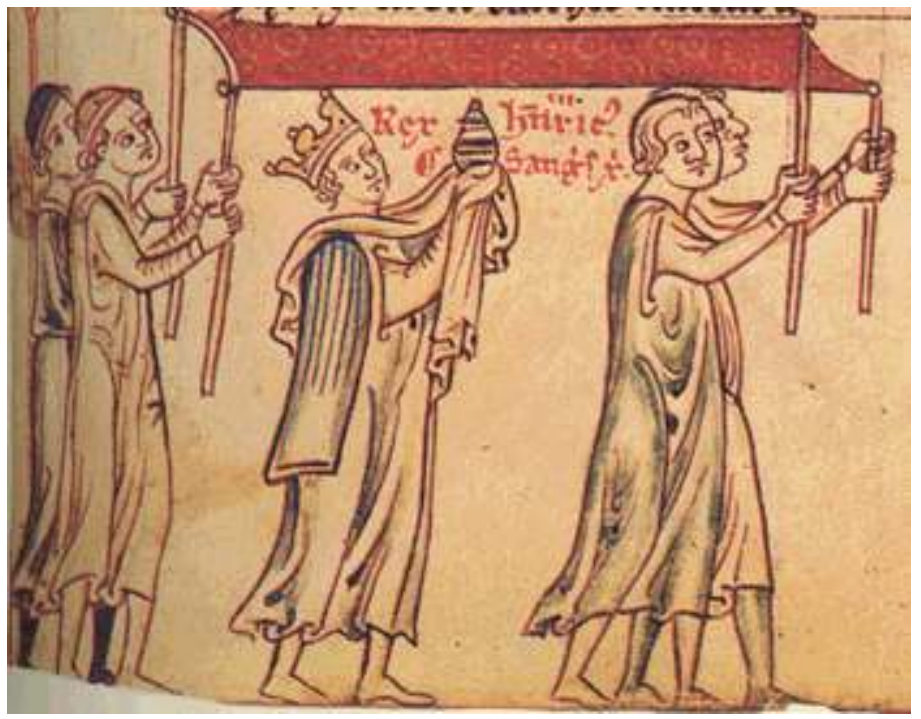

Ostension du saint Sang de Westminster par Henri III, c.1250, Chronica Majora de Mathieu Paris, Corpus Christi College, Cambridge, MS 16 II, f. 216r.

Wikimedia Commons.

Comment l'officiant manipule-t-il la relique? Francesca Sbardella livre une ethnographie du traitement des reliques dans des couvents augustins et carmélites. La manipulation s'y caractérise par des gestes et des séquences de gestes standardisées et un «savoir des mains» que l'autrice rapproche d'une "gestualité des savoirs techniques ${ }^{67} »$. Une telle finesse d'analyse nous est interdite, car nos sources sont rarement précises, et nous devons nous contenter d'un départ entre de grands types de gestes.

Parmi eux, on pense bien sûr au geste d'élévation, rapprochant les reliques de l'eucharistie ${ }^{68}$. On le rencontre quelquefois, Jacques Le Saige parle ainsi de l'« élévation » d'une image à Lorette (J.L., p. 33). L'iconographie travaille rarement dans ce sens et reste conforme le plus souvent à l'étymologie de l'ostension, os-tendere, tendre en avant : on tend la relique non pas vers le ciel, mais vers la foule. Ainsi, dans une enluminure du guide pour les pèlerins de Rome, les Mirabilia Urbis Romae, la Véronique est montrée en hauteur, depuis la chaire de Saint-Pierre (fig. 1).

Le geste se fait alors plus volontiers descendant ${ }^{69}$, comme à Aix, où la chemise se déplie vers les fidèles au pied de l'église, ou encore à Düren, où Philippe décrit l'ostension du crâne de sainte Anne :

[on montre] au peuple qui est en bas cette sainte tête et le prélat qui la tient la retourne, sens dessus dessous, pour montrer le sommet de la tête toute nue, et il est garni d'argent et sur la tête il y un petit plateau qui se lève, et alors il semblait que tout allait se fendre à force de jouer de la corne et de la busine [instrument à cuivre], tellement que l'on pleurait quasiment de joie (P.V., p. 179) ${ }^{70}$.

Si on a bien compris la description de Philippe, le renversement du crâne serti d'argent déclenche par gravité l'ouverture d'un couvercle dévoilant l'os, de sorte que comme à Aix, la relique s'ouvre toute seule. Ce geste renversant ponctue une longue séquence de gestes le préparant, avant la séquence inverse, replier, renfermer, verrouiller, repartir : l'ostension se présente comme le dépliage d'un ample et fastueux dispositif dont cette petite surface d'os est la pointe. 


$$
\begin{aligned}
& \text { Chapelle, Philippe raconte qu'au moment où la chemise se déplie, alors que le soleil est } \\
& \text { à son zénith, des témoins voient une étoile dans le ciel (P.V., p. 176), manifestant cette } \\
& \text { puissance sur-matérielle de l'acte de montrer. }
\end{aligned}
$$

\section{Qui ne pleure pas n'est pas chrétien}

En ouverture de son article sur les processions parisiennes flamboyantes, Jacques Chiffoleau interroge la "progressive théâtralisation des processions»: «cette théâtralisation, cette esthétisation du rituel, si sensible à la lecture même des chroniqueurs, implique en effet, me semble-t-il, la transformation de certains participants en spectateurs, c'est-à-dire la transformation de leur statut même de sujets qui 'voient' désormais, bien plus qu'ils ne 'font" ${ }^{\prime 71}$." "À la limite, tous les processionnaires deviennent spectateurs de leurs propres gestes » reprend plus loin Jacques Chiffoleau ${ }^{72}$.

60 Nos témoins voient, c'est indéniable, mais il est tout aussi indéniable qu'en voyant, ils font. Les textes s'attardent régulièrement sur la description de l'affluence des fidèles, élément qui manifeste l'importance de la relique, ainsi que sur leurs attitudes et leurs réactions.

61 «Le peuple est à genoux, la tête découverte et les mains jointes (P.V., p. 176) », d'après Philippe de Vigneulles : c'est la position de "génuflexion ${ }^{73}$ » que prennent aussi les fidèles assistant à l'ostension de la Véronique (fig. 1). Mais les fidèles ne restent pas figés dans une posture déférente : à la fin du rituel, quand la chemise se déplie ou que le crâne s'ouvre, Philippe décrit les pleurs, les cris de la foule et le son des cornes. Il ne s'agit pas uniquement des instruments des «ménestrels de la ville»: «tout le peuple jouait de leurs cornets » écrit-il (P.V., p. 173). » La foule participe activement à la puissance de cette épiphanie en manifestant son émotion, en criant ou en soufflant dans des cornes ; sa présence et ses réactions sont une fois encore ornementales.

Celles-ci peuvent prendre un tour qui déplaît au clergé, par exemple lorsque les fidèles jettent sur la relique des objets afin de les rapporter chargés de virtus. Lors de l'ostension du Sacro Catino de Gênes, vase dans lequel le Christ et ses disciples auraient mangé, Antonio de Beatis raconte la défense lue aux fidèles sous peine de mort de jeter des objets sur l'armoire en verre contenant le vase, de peur de le briser (A.B., p. 281). On retrouve des statuts comparables à la fin $\mathrm{du}_{\mathrm{XVI}} \mathrm{e}^{\mathrm{e}}$ et au début du XVII ${ }^{\mathrm{e}}$ siècle pour les ostensions du saint Suaire de Besançon, montré «le plus expéditivement qu'il est possible, afin de laisser la dévotion en la maîtrise des assistants, là où si la chose est prolongée, la dévotion se perd et la confusion arrive, en raison des chapelets que l'on jette contre le saint linge pour les faire toucher, et du tumulte qui s'en suit ${ }^{74}$. »

63 Cette source tardive fait affleurer la question, absente de notre documentation, de la durée de la monstration, de la bonne maîtrise du rythme dans lequel s'enchaînent les gestes qui composent le rituel. Elle témoigne encore du souci des officiants de contrôler une émotion dévote débordante. Pour autant, il est normal que l'émotion dévote soit à la mesure de la puissance extraordinaire de la relique : il faut donc encadrer ce moment d'excès.

64 Il y a bien une injonction sociale d'être ému par la relique : Jean Gallot, décrivant le voyage des frères de Clairvaux à Rome, en 1520, et visitant le saint Suaire à Chambéry,

Images Re-vues, 16 | 2019 
écrit : « il n'est pas chrétien à mon avis celui qui, en le voyant, n'a pas de crainte en lui et ne se tait pas $^{75}$. " On ajoute donc la crainte devant la puissance de la relique aux autres réactions émotionnelles qu'elle provoque. Le fidèle participant à l'émotion collective s'affirme comme partie prenante d'un corps social solidaire, celui qui n'est pas ému est exclu de cette « communauté émotionnelle ${ }^{76}$ ».

C'est le sens de notre obstination à qualifier d'ornemental tout ce et tous ceux qui entourent la relique dans son exposition; la mise en visibilité de la relique met en visibilité le monde qui l'entoure. Avec la relique, la communauté s'expose à elle-même, en une représentation collective ordonnée où chacun se trouve d'une part situé socialement par rapport à la relique, mais encore amplifié par sa participation active à l'ornementalisation de la relique.

\section{L'ostension faite image}

Parmi les images de pèlerinage, les enseignes de plomb présentent un grand intérêt. Vendues dans les sanctuaires, elles étaient rapportées par les pèlerins. La documentation ne permet pas de dire précisément à quels types de pratiques elles donnaient lieu ${ }^{77}$, mais on sait que les fidèles faisaient toucher leurs enseignes aux reliques pour récupérer un peu de leur virtus, leur énergie miraculeuse. Leur iconographie vise à exprimer, sur une petite surface, l'identité visuelle de la relique ${ }^{78}$.

Parmi ces solutions visuelles, la plupart des enseignes représentent le saint dont les restes sont conservés, avec ses attributs, ou dans une scène significative de son hagiographie. Certaines jouent avec l'aspect visuel de la relique ou du reliquaire: le visage du Christ suffit à évoquer la sainte Face de Véronique conservée à Rome, les fragments de croix, les saintes maisons de la Vierge, donnent lieu à des enseignes en forme de croix et de petites maisons, le sanctuaire de Wilsnack produit des enseignes en forme trilobées pour rappeler les trois hosties sanglantes conservées dans l'église.

On trouve aussi un groupe d'enseignes figurant la monstration de la relique. C'est le cas pour la tunique de la Vierge d'Aix-la-Chapelle ou du saint Suaire de Chambéry-Turin, dont les enseignes représentent les officiants montrant l'objet (fig. 6 et 7). C'est un type d'iconographie qu'on retrouve aussi dans les gravures des XIV ${ }^{\mathrm{e}}-\mathrm{XVI}{ }^{\mathrm{e}}$ siècles, notamment dans les livrets de pèlerinages ${ }^{79}$, et qui semble associé aux reliques donnant lieu à de grandes ostensions à dates fixes, comme dans le cas des ostensions publiques d'Aix-laChapelle et de Nuremberg, mais aussi celles des linges imprimés de la Véronique à Rome et du saint Suaire à Chambéry puis à Turin; il ne semble pas excessif de parler pour ces reliques de "reliques à monstration", leur identité étant chevillée à ce dispositif. 


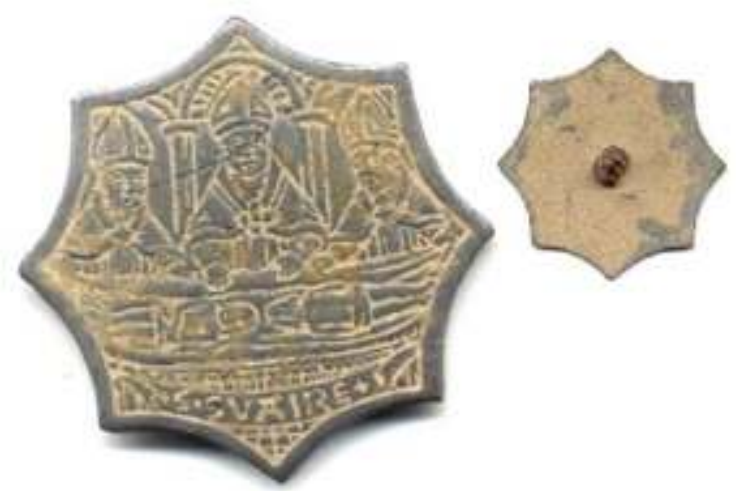

Enseigne de pèlerinage du saint Suaire de Chambéry, xvi siècle, médaille mentionnée par un amateur en ligne.

http://www.la-detection.com/dp/message-4404.htm.

Figure 7

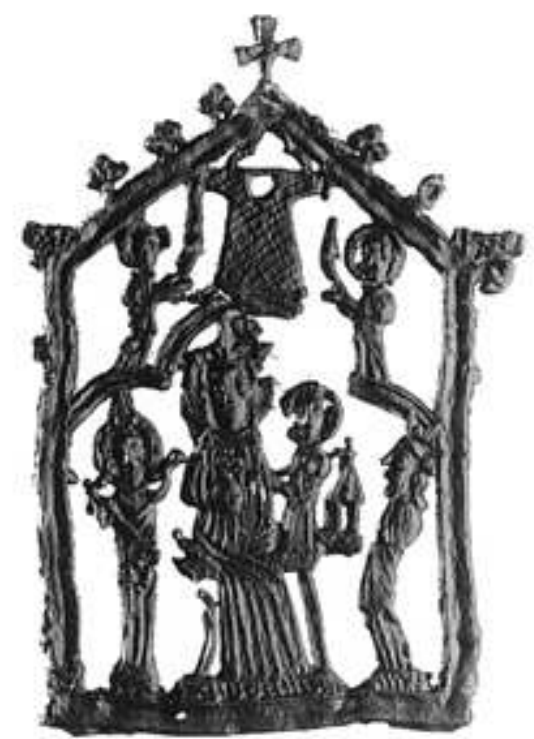

Enseigne de pèlerinage d'Aix-la-Chapelle figurant Marie, saints Corneille et Charlemagne, sous

l'ostension de la Tunique de la Vierge, xive siècle, plomb, trouvée à Dordrecht, collection van Beuningen, $n^{\circ} 0381$.

D'après http://kunera.nl/.

S’il semble assez logique que les "images-reliques » soient bien représentées dans ce groupe, on est frappé par la prégnance de reliques textiles : ainsi la plupart des suaires du Christ, imagés ou non, font l'objet d'ostensions, à Compiègne, Cadouin, Toulouse, Aix-la-Chapelle, Chambéry. S'il est encore trop tôt pour expliquer ce phénomène, la dialectique du dévoilement d'une part et les jeux liturgiques de Pâques dans lesquels des clercs jouant les saintes Femmes montraient le saint Suaire aux fidèles semblent deux pistes intéressantes pour comprendre cette surreprésentation.

Le saint Suaire de Chambéry-Turin est une de ces reliques ; parmi la quinzaine d'images qui le représentent, au Xvi ${ }^{\mathrm{e}}$ siècle, presque toutes le figurent montré par des anges ou des clercs. Plus précisément, on représente les trois évêques qui procédaient aux 
ostensions savoyardes lors de la fête du saint Suaire, le 4 mai (fig. 6 et 8). Dans la décennie 1520, lorsque le saint Suaire de Besançon apparait, probable copie de l'image de Chambéry, ses promoteurs cherchent non seulement à en copier l'image, mais aussi le dispositif ornemental: le nombre des officiants et le calendrier des ostensions se rapprochent $\mathrm{du}$ dispositif savoyard. La relique est indissociable de son régime d'ostension, de sorte que pour assurer l'efficace du suaire de Besançon, il fallait copier non seulement l'image, mais aussi les ostensions de Chambéry.

Figure 8

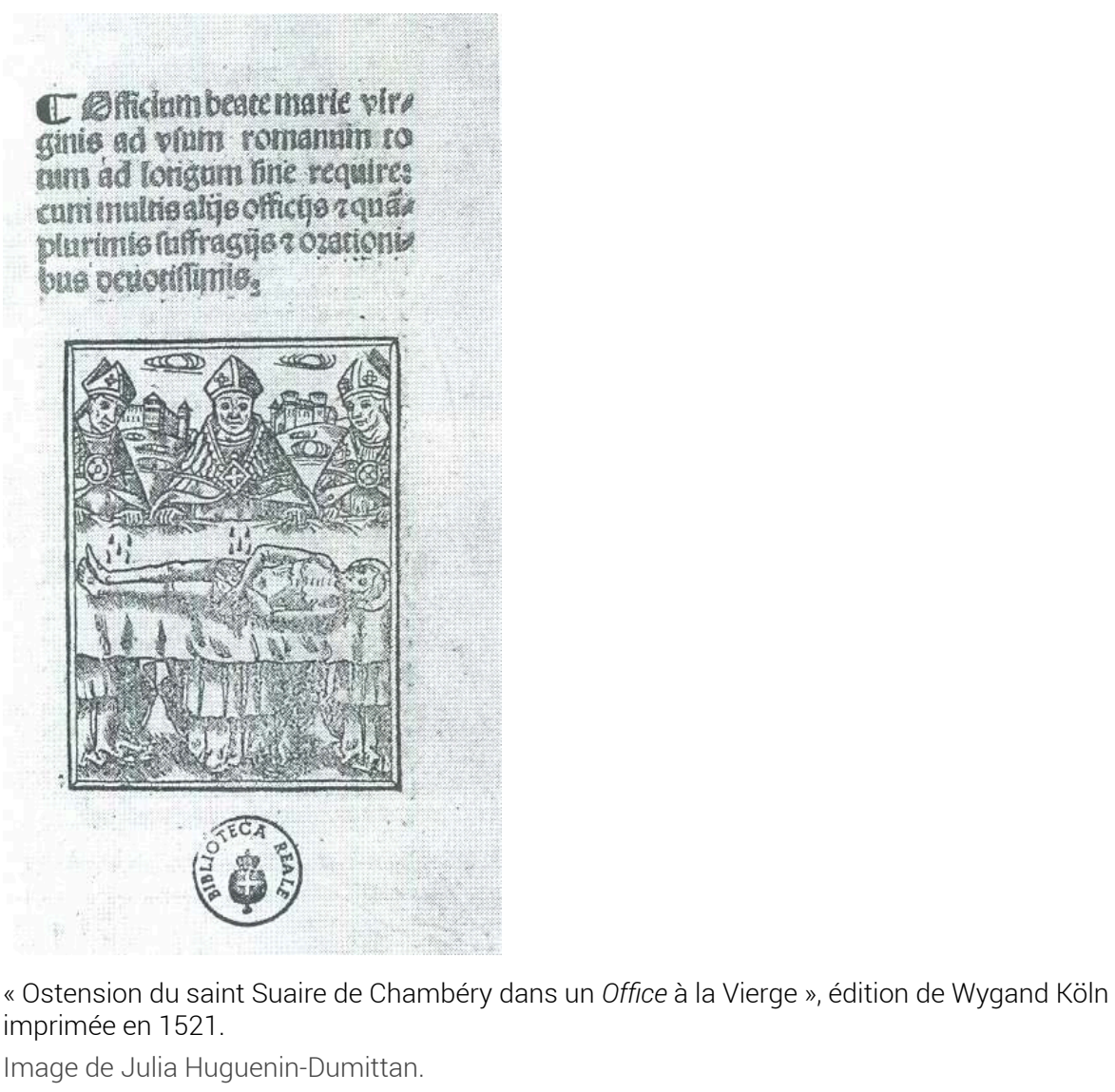

71 On voit ici l'importance du dispositif d'ostension dans la construction du pouvoir de la relique; le pèlerin qui porte l'enseigne du pèlerinage de Chambéry sur laquelle on fait ostension du saint Suaire redouble son exposition. Les enseignes redoublent parfois tout le dispositif, le vêtement des officiants, les luminaires, les architectures voire même les fidèles, mais peuvent aussi se résumer à la représentation d'un clerc ou d'un ange qui expose le corps saint, isolant l'efficacité du geste de montrer.

La substitution d'un ange à l'officiant est fréquente pour les reliques à monstration : ainsi le frontispice d'un office pour la fête du saint Suaire de Chambéry au début du XvI ${ }^{\mathrm{e}}$ siècle présente le drap porté par un ange (fig. 9), iconographie que l'on retrouve sur une médaille figurant la tunique de la Vierge d'Aix-la-Chapelle (fig. 10). Les anges sont omniprésents et polysémiques dans les images médiévales, ils sont notamment vêtus comme des officiants portant des accessoires liturgiques, lampes, livres, encensoirs. On les retrouve régulièrement montrant les Instruments de la Passion. Maurice McNamee a étudié cette iconographie et l'a reliée au pouvoir sacramentel des clercs et à 
l'eucharistie ${ }^{80}$. En figurant ce geste de monstration réalisé par un ange vêtu en clerc, on soulignerait donc le pouvoir sacramentel du geste de monstration et la parenté entre le corps du saint et le plus saint des corps.

Figure 9

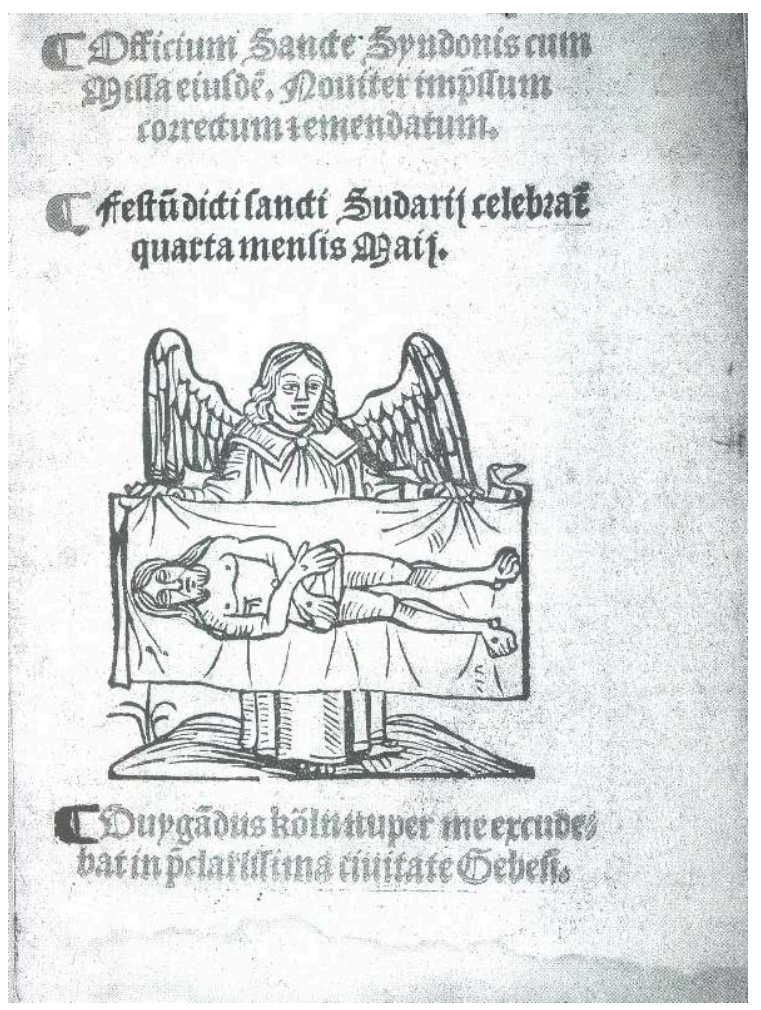

«Le saint Suaire montré par un ange », gravure imprimée d'abord à Genève par Wygand Köln, ici reprise dans un office pour la Vierge imprimé par Antoine Ranoto à Turin en 1531, Turin, Biblioteca Reale.

Image de Julia Huguenin-Dumittan. 
Figure 10

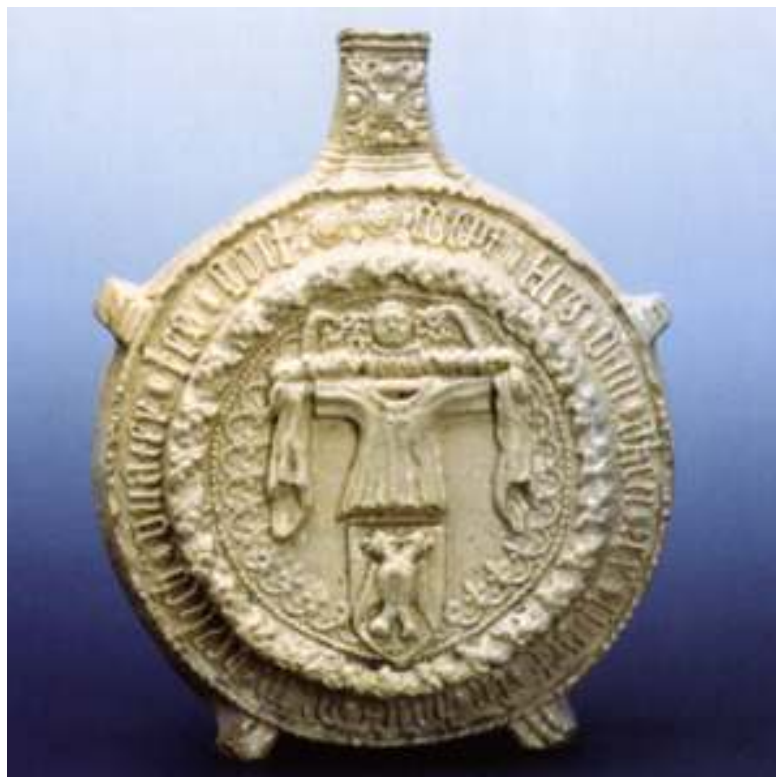

Enseigne de pèlerinage d'Aix-la-Chapelle, figurant un ange portant la tunique de la Vierge, c.1500, Dortmund, Museum der Stadt, $n^{\circ} \mathrm{C} 5442$

D'après http://kunera.nl/.

Dans une autre dimension, les anges porteurs d'objets sacrés, mais aussi porteurs de l'âme des défunts incarnent une dialectique du mouvement vertical ciel/terre, déposition/élévation. L'ange descend sur terre pour y manifester la présence céleste et accompagne aux cieux les ressuscités.

Une clé du cloître de Cadouin datant de la fin du $\mathrm{xv}^{\mathrm{e}}$ siècle ${ }^{81}$ surprend le visiteur contemporain (fig. 11). Celui-ci aura vu le fac-simile du saint Suaire conservé dans l'abbaye, une riche étoffe brodée, mais sans image. Pourtant, ici, quatre anges tiennent un linge sur lequel se détache le corps du Christ. La clé représente-t-elle le sacré drap de Cadouin ? Il semble bien, mais le sculpteur a choisi de mettre en image la présence $\mathrm{du}$ Christ sur la toile aniconique de la relique. Les trois anges sont-ils en train de transporter le Christ supplicié aux Cieux, enveloppé dans le saint Suaire, sorte d'Ascension, ou bien exposent-ils aux visiteurs en contrebas le saint linge conservé dans l'abbaye, sorte d'ostension? La bidirectionnalité proposée par cette clé sculptée signale la charge épiphanique des ostensions qui se pratiquaient alors à Cadouin; elles sont la descente du sacré, depuis le ciel vers les fidèles, depuis l'espace sacré du chœur vers le siècle; elles préparent la conversion et le Salut des fidèles, regards tournés vers le chœur et vers le ciel. 
Figure 11

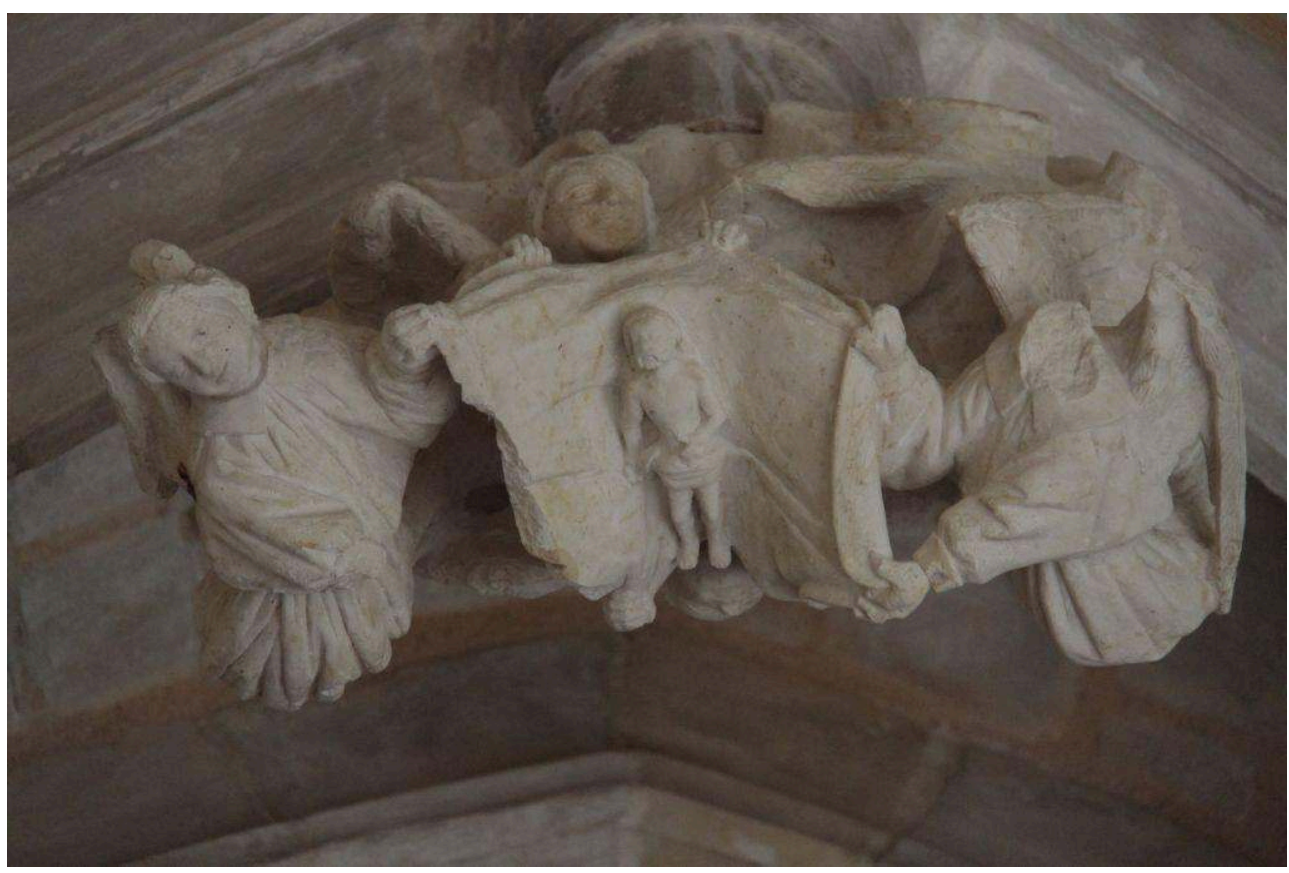

Anges portant le saint Suaire, clé du cloître de l'abbaye de Cadouin, c.1495.

Image Danielle Martinez-Michelle Fournié.

\section{Conclusion}

Un linge déployé, taché du sang d'un saint ou immaculé, un crâne serti d'or ou un tibia dans un reliquaire à monstrance sont autant de corps-images, étant donné le soin particulier avec lequel on organise leur visibilité. Les multiples descriptions de monstrations de reliques, rythmant les récits de pèlerins, et la précision de ces descriptions, signalent l'importance de ces pratiques par ailleurs mal renseignées par les sources canoniques et liturgiques ; elles sont fréquentes et elles sont recherchées, par les pèlerins comme par les paroissiens. On en trouve un large nuancier, des spectaculaires épiphanies publiques célébrées ponctuellement dans les sanctuaires rhénans aux visites de cryptes, menées par un clerc dont l'épais trousseau permet d'accéder au plus près du saint.

Nos témoins disent régulièrement avoir vu la relique « nue », le contact visuel de l'œil $\mathrm{du}$ fidèle avec le corps saint a donc une efficacité particulière. Jacques Lesaige qualifie les corps entassés des saints Innocents de Murano comme « quelque chose de beau »: le terme est fréquent en moyen français, il peut renvoyer à un jugement proprement esthétique ou plus général, "remarquable ", "plaisant». Il semble bien s'agir d'un rapport visuel plaisant et dévot à la relique. Dans cette enquête, nous n'avons pas isolé l'acte de voir directement la relique, cette question de la beauté propre du corps-image qu'est la relique reste donc ouverte. Il faut toutefois souligner que la description de la relique elle-même est assez rare dans ce corpus et prend le plus souvent moins de place que la description des lieux, des gestes et des objets qui l'accompagnent. Le corps saint est donc inséparable du dispositif ornemental qui le prolonge et qui amplifie son efficacité symbolique et sa puissance d'image. 
77 Lorsqu'on montre la relique, elle est entourée d'objets, mais aussi d'acteurs en mouvement, dont la gestualité contribue à animer la relique. Parmi ces acteurs, il faut considérer aussi bien ceux qui montrent que ceux qui voient et s'émeuvent. Ainsi, les participants des monstrations se donnent à voir avec les reliques, les décorant autant qu'ils sont décorés par elles. De la sorte, en hiérarchisant l'accès des fidèles aux reliques et en mettant en ordre l'assemblée des officiants et des dévots lors des monstrations, le corps social se montre à lui-même avec le corps saint.

\section{NOTES}

1. «Le 23 de may nous allasmes juer jusqua la ville de Moran, laquelle est une petite ville, ou il y a des belles maisons. [...] On y faict tant de voir de cristale que nul ne le croiroit s'il ny estoit. Je en vis faire de toute sorte. Après les avoir veu faire, allasmes ouyr messe en lune des eglises. Quant le prestre eult dit messe on luy apporta des clefs. Adoncq haulcha ledit autel, ou il avoit dit messe, comme se eust esté ung coffre et nous monstra dedens deux cens et huit corps de petits innocens saincts, et le plus part avoient encoire leurs chemisettes : et estoient entassés par licts dedens ledict autel, dont c'est quelque chose de beau. Nous en fusmes tous resjouis de veoir tant de sy beaux josnes saincts. Après revinsmes a nostre nef. Et adoncq retournasmes disner a Venise. Mais soiez seur que j'avoie bon apetit. » Pour la référence, voir les abréviations note $n^{\circ} 6$.

2. Pour une approche anthropologique de cette question lire Jean-Claude schмiтt, Le corps des images, Paris, Gallimard, 2002, ainsi que Caroline W. BYnum, Christian Materiality, An Essay on Religion in Late Medieval Europa, New York, Zone Book, 2015.

3. Sur la diversité des formes d'autel lire Joël PERRIN, « L'autel : fonctions, formes et éléments ", dans In Situ, $\mathrm{n}^{\circ}$ 1, 2001, http://journals.openedition.org/insitu/1049 (consulté le 28 mai 2019) : il évoque dans sa typologie l' "autel-coffre ", note $n^{\circ} 119$, souvent en bois. On connait des autels dont une partie ouverte ou mobile sur la face antérieure laisse voir les reliques (les autelsconfessions et expositions, n.5 et \$89), mais l'ouverture sommitale décrite ici est plus singulière. L'autel de San Pietro Martire de Murano ne nous est malheureusement pas conservé, l'église ayant été rebâtie.

4. Sur le genre du récit de pèlerinage, une bibliographie a été proposée par Magali CHEYNET, «La route des pèlerins, éléments bibliographique », Questes, $\mathrm{n}^{\circ} 22$, 2011, p. 97-104, https:// journals.openedition.org/questes/1465 (consulté le 5/08/2019). Pour un aperçu général du pèlerinage médiéval et son historiographie lire Catherine VINCENT, « Du nouveau sur le pèlerinage médiéval ? ", dans Sophie CASSAGNES-BROUQUET, Amaury CHAUOU, Daniel PICHOT et Lionel ROUSSELOT (dir.), Religion et mentalités au Moyen Âge : Mélanges en l'honneur d'Hervé Martin, Rennes, Presses Universitaires de Rennes, 2003, p. 379-386, http://books.openedition.org/pur/19838 (consulté le 5/08/2019).

5. Ces bornes chronologiques délimitent une étape importante dans l'affirmation d'une culture de pèlerinage, avant les bouleversements engendrés par la Réforme. Philippe Cordez les emploie et souligne leur importance notamment pour le développement des livrets de pèlerinage : Philippe CORDEZ, «Wallfahrt und Medienwettbewerb. Serialität und Formenwandel der Heiltumsverzeichnisse mit Reliquienbildern im Heiligen Römischen Reich (1460-1520)», dans Andreas TACKE (dir.), "„Ich armer sundiger Mensch“. Heiligen- und Reliquienkult am Übergang zum konfessionellen Zeitalter ", Göttingen, Wallstein-Verlag, 2006, p. 37-73. 
6. Une telle enquête est permise par l'ample travail d'édition de ce type de récits, réalisé notamment en France autour de Denise Péricard-Méa. Il est facilité par la mise en ligne de la plupart de ces textes, pour cette bibliographie je privilégie ces éditions accessibles en ligne. Je ne retranscris le texte original en note que lorsque j'en propose une traduction :

Léon DE ROzMITAL, «Itinéraire (1465-1467)», publié dans De la Bohême jusqu'à Compostelle. Aux sources de l'idée d'union européenne, Denise Péricard-Méa (éd.), Biarritz, Atlantica, 2008 (ci après L.R.).

Hans von WALTHEYM, «Le pèlerinage en l'an 1474 », publié dans Provence historique, Annie Faugère (trad.), t.41, 166, 1991, p. 465-551 (ci après H.W.). Le fascicule est en ligne, URL : http://provencehistorique.mmsh.univ-aix.fr/n/1991/Pages/Fasc-166.aspx. Le texte original est aussi en ligne, Die Pilgerfahrt, Friedrich Emil Welti (dir.), Bern, Stämpfli, 1925, URL: https://www.nvf.ch/pdf/ waldheim_welti.pdf.

Felix FABRI, «Les errances (1480-1483) », publié dans Les errances de Frère Félix, pélerin en Terre sainte, en Égypte et en Arabie, Nicole Chareyon et Jean Meyrs (éd. et trad.), Montpellier, CERCAM, 2000 (ci après F.F.).

Jean DE TOURNAI, «Le voyage (1488-1489) " publié dans «Complément au récit de voyage publié aux éditions La Louve, Le Voyage de Jean de Tournai. De Valenciennes à Rome, Jérusalem et Compostelle", Fanny Blanchet-Broekaert (éd.), dans Saint-Jacques Info, http:// lodel.irevues.inist.fr/saintjacquesinfo/index.php?id=1567, consulté le 20/05/2019 (ci après J.T.). Jérôme MÜNZER, «Itinéraire (1495) » publié dans «L'itinéraire de Jérôme Münzer », Paul Amargier et Jean-Louis Charlet (trad.), dans Provence historique, t. 41, 166, 1991, p. 586-599 (ci après J.M.). Le fascicule est en ligne, URL: http://provence-historique.mmsh.univ-aix.fr/n/1991/Pages/ Fasc-166.aspx. Pour les passages hors de Provence j'utilise l'édition de Denise Péricard-Méa, De Nuremberg à Grenade et Compostelle. Itinéraire d'un médecin allemand, Biarritz, Atlantica, 2009 (ciaprès J.M.')

Antonio DE BEATIS, «Voyage (1517-1518)», publié dans Voyage du cardinal d'Aragon, Madeleine Havard de la Montagne (trad.), Paris, Perrin, 1913, URL: https://gallica.bnf.fr/ark:/12148/ bpt6k110458d (ci après A.B.). Le texte original est aussi en ligne, Die reise des kardinals Luigi d'Aragona, Freiburg-im-Breisgau, Herdersche v., 1905, URL: https://archive.org/details/ diereisedeskard00pastgoog/page/n12.

Philippe DE VIGNEULLES, «Chronique (1510) », publié dans Gedenkbuch des Metzer Bürgers Philippe von Vigneulles, Henri-Victor Michelant (éd.), Stuttgart, Gedruckt auf Kosten des Vereins, 1852, URL : https://archive.org/details/bub_gb_wq0LAAAAIAAJ (ci après P.V.).

Jacques LESAIGE, "Voyage (1518)», publié dans Voyage de Jacques Lesaige, Romain-Hyppolite Duthilloeul (éd.), Douai, Adam d'Aubers, 1851, URL: https:/gallica.bnf.fr/ark:/12148/ bpt6k1061216?rk=21459;2, consultés le 22/08/2019 (ci après J.L.).

Cette enquête a bénéficié de la lecture et des précieux conseils de Philippe Cordez, Pierre-Olivier Dittmar, Didier Méhu, Alain Rauwel et Jean-Claude Schmitt. Je les en remercie vivement.

7. "Là nous fut monstré le chief de sainct Jacques (J.T., f. 291) ».

8. Denise PÉRICARD-MÉA, Compostelle et cultes de saint Jacques au Moyen Âge, Paris, PUF, 2000, p. 98-103.

9. «Il y avoit dedens ladicte cappelle ung des officiers de ladicte église lequel avoit une robe moitié blance et l'aultre moictié vermeille lequel dict en trois langaiges, c'est à scavoir en latyn, en allemant et en franchois et le dict tout hault, c'estoit que quiconques ne croioit fermement que le corpz de sainct Jacques ne soit encassé ou machonné dedens le grand autel de ladicte église et aussy comme il appert par ladicte lettre au rollet laquelle ensaigne l'ymaige quy est deseure le grant autel auquel comme dict est, est escript en lettres romaine Hic jacet ..., il dict qu'il faict son pèlerinaige en vain. » 
10. On pense aux travaux pionniers de Carol HEITZ, Recherches sur les rapports entre architecture et liturgie à l'époque carolingienne, Paris, SEVPEN, 1963. Lire aussi Paolo PIVA (dir.), Art médiéval. Les voies de l'espace liturgique, Paris, Picard, 2008, et tout particulièrement son article sur la question de l'architecture de pèlerinage : Paolo PIVA, « Le déambulatoire et les 'trajets' de pèlerinage dans les églises d'Occident (Xe-XIIe siècle) », dans Paolo PIVA (dir.), Art médiéval, op. cit., p. 80-129.

11. Pour décrire les grandes cérémonies liturgiques, la documentation latine utilise le plus souvent le terme d'«ostensiones", le moyen-français d' "ostensions » et de "monstres de reliques"; les pèlerins voyant une relique emploient généralement le passif "on nous a monstré ». Je choisis donc le terme de "monstration », qui intègre toutes les pratiques donnant à voir les reliques, mêmes informelles, par exemple une visite de crypte, pour réserver le terme d'« ostensions » aux grandes cérémonies liturgiques.

12. Jean-Claude schmITT, Le corps des images, op.cit. et Jérôme BASCHET, L'iconographie médiévale, Paris, Gallimard, 2008, notamment sur l'« image-objet » p. 22-62.

13. Pierre-Olivier DITTMAR, «Cachez ce saint que je ne saurais voir. Modifications de visibilité en contexte rituel à la fin du Moyen Age », Cahiers d'anthropologie sociale, n 11, 2015, p. 84-99.

14. Philippe CORDEZ, «Les reliques, un champ de recherches. Problèmes anciens et nouvelles perspectives ", Bulletin d'information de la Mission Historique Française en Allemagne, $n^{\circ} 43,2007$, p. 102-116.

15. Voir dans Giuseppe ALBERIGo (éd. et trad.), Les conciles oecuméniques, II, Paris, Cerf, 1994, p. 560-561. Le canon est issu d'un des décrets du synode de Mayence de 813, inséré dans le Décret de Gratien, André vauchez La Sainteté en Occident aux derniers siècles du Moye-Âge. D'après les procès de canonisation et les documents hagiographiques, Rome, École française de Rome, 1988, p. 33. Pour la discussion du sens de ce canon lire notamment Hartmut KÜHNE, Ostensio Reliquarium, Untersuchungen über Entstehung, Ausbreitung, Gestalt und Funktion der Heiltumsweisungen im römischdeutschen Regnum, Berlin-New York, De Gruyter, 2000, p. 555-563 et Philippe CORDEZ, Trésor, merveille, mémoire. Les objets des églises au Moyen Âge, Paris, Éditions de l'EHESS, 2016 p. 50.

16. Étienne ANHEIM, Isabelle HEULLANT-DONAT, Emmanuelle LOPEZ, Odile REDON, « Rome et les jubilés du XIV $\mathrm{X}^{\mathrm{e}}$ siècle : histoires immédiates ", Médiévales, $\mathrm{n}^{\circ}$ 40, 2001, p. 53-82, notamment p. 67-68 pour ce qui concerne la Véronique.

17. Dans son impressionnante et précieuse somme Ostensio Reliquiarum, op.cit., Hartmut Kühne fait la liste des sources pour le mode d'ostension qu'il décrit, les ostensions publiques allemandes de la fin du Moyen Âge, ch. "Die Quellen», p. 31-79. Des sources liturgiques décrivant les ostensions existent, mais elles sont locales (p. 51-55).

18. C'est notamment la thèse de Roland RECHT, Le croire et le voir. L'art des cathédrales, $\mathrm{XII}^{\mathrm{e}}-\mathrm{XV}^{\mathrm{e}}$ siècle, Paris, Gallimard, 1999, p. 99-103.

19. Gia toussaint, Kreuz und Knochen : Reliquien zur Zeit der Kreuzzüge, Berlin, Reimer, 2011, l'autrice réfute cette thèse d'une dévotion visuelle proprement gothique apparue au XIII ${ }^{\mathrm{e}}$ siècle, p. 15-23. Sur les monstrances et la visibilité des reliques, lire aussi Pierre-Olivier DITTMAR, «Cachez ce saint ", op. cit.

20. Hartmut KÜHNE, op. cit., p. 552. Le texte de 1079 évoque l'ostensio du saint Suaire à l'occasion d'une translatio d'un reliquaire à un autre, la documentation plus tardive décrit la foire autour de l'ostension du saint Suaire mais ne nous dit rien de précis sur sa liturgie; Nicolas SARzEAUD, "Saint Suaire de Saint-Corneille de Compiègne », Inventaire des sanctuaires et lieux de pèlerinage chrétiens en France, http://sanctuaires.aibl.fr/fiche/785/saint-suaire-de-saint-corneille-decompiegne, version du 14/10/2018, (consulté le 29/05/2019).

21. Sur le développement de ces fêtes lire Charles MERIAUX, «Fêtes et jours chômés dans le monde carolingien (VIII ${ }^{\mathrm{e}}-\mathrm{x}^{\mathrm{e}}$ siècles) », dans Philippe DESMETTE et Philippe MARTIN (dir.), Orare aut laborare? Fêtes de précepte et jours chômés du Moyen Âge au début du XIX ${ }^{e}$ siècle, Villeneuve d'Ascq, Presses 
Universitaires du Septentrion, 2017, p. 51-52, lire encore une fois Hartmut KÜHNE, op. cit., pour les pratiques ayant précédé l'ostensio p. 520-534.

22. Carol HEITZ, op. cit.

23. Georgie FRANK, The Memory of the Eyes: Pilgrims to Living Saints in Christian Late Antiquity, Berkeley, University of California Press, 2000.

24. Ce n'est pas toujours évident; Jean de Tournai cherche l'icône de la Vierge de Philerme à Rhodes et s'enquiert du chemin auprès d'un clerc grec ne parlant pas latin ; s'en suit un dialogue de signes, où Jean montre l'image de la Vierge dans son livre d'Heures et où le clerc grec mime le chemin qui lui permet finalement de trouver l'image (J.T., f. 234v).

25. Jean de Tournai, f. 282, mentionne l'implication de son hôte dans la visite de Saint-Sernin de Toulouse et Philippe de Vigneulles, p. 182, d'un guide dans sa visite de Cologne. Sur le cas de Saint-Sernin que je n'ai pas traité ici et que visite aussi Jérôme Munzer, lire SOPHIE BROUQUET, MiCHELLE fourniÉ, "Le Saint des Saints: le Trésor de Saint-Sernin de Toulouse », dans MICHELLE FOURNIÉ, DANIEL LE BLÉVEC, CATHERINE VINCENT (dir.), Corps saints et reliques dans le Midi, XII ${ }^{e}$-XVe siècles, Cahiers de Fanjeaux, $n^{\circ}$ 53, 2018, p. 205-265 et notamment p. 231-234 sur l'accès des pèlerins aux reliques. Les recherches du groupe de recherche toulousain sur les reliques de saint Jacques à Sernin se poursuivent et nous en attendons avec impatience les nouveaux résultats. Je remercie Michelle Fournié pour son aide précieuse sur les dossiers de reliques toulousaines.

26. Le rôle des patrons est décrit par Felix Fabri, lire Jean MEYERS, « L'encadrement du pèlerin de Jérusalem à la fin du XV siècle d'après l'Evagatorium de Frère Félix Fabri ", dans Jean-Marie CAUCHIES (dir.), L'encadrement des pèlerins du XII siècle à nos jours, Bruxelles, Fac. Univ. Saint-Louis, 2010, p. 125-152.

27. "Mais on ne nous monstrait nulle relicque, car il les monstrent en d'autres temps et saixons $"$.

28. Plusieurs de nos pèlerins décrivent leurs expériences de ce reliquaire largement étudié (A.B., p. 104-105, L.R., p. 148-149, J.T., f. 5), lire par ex. Lisa V. CIRESI, « A liturgical study of the shrine of the Three Kings in Cologne ", dans Colum HOURIHANE (dir.) Objects, images and the Word: art in the service of the liturgy, Princeton University Press, 2003, p. 202-230.

29. La pratique n'est pas proprement provençale, on la retrouve pour les suaires de Toulouse, Chambéry et Besançon par exemple, voir les documents cités note $n^{\circ} 36$.

30. «En tout le pays de Provence on ne peult voir nulz sanctuaires sans avoir avec les thésauriers des églises ung homme de la justice des villes car comme vous diries le prévost ou eschevin des villes ont une clef des thésaures [...]. »

31. Sur la charge de trésorier, lire Philippe CORDEZ, Trésor, mémoire, merveilles, op. cit. Il délimite notamment la fonction du trésorier p. 38-46.

32. Jean souhaitait visiter trois sanctuaires provençaux (Saintes-Maries de la Mer, Saint-Louis d'Arles et Saint-Gilles-du-Gard») mais raconte : «je n'osai pas y aller parce qu'il fallait payer, selon la coutume, dix pièces d'or chacune, et je ne refusai pas tant pour la dépense et la perte d'or ou d'argent, mais parce que souvent on est examiné mais on ne sait pas par qui et il n'y a pas besoin que les étrangers sachent les choses que l'on porte et si on a de l'argent ou pas. La coutume est fort mauvaise et dangereuse, c'est assez pour meurtrir les gens [...] (Nous nous partismes dudict Tarrascon le lundy XVe dudict mois pour aller aux Trois Maries, à Sainct Loys d'Alle et à Sainct Gilles lesquelz corpz sainctz je fusse volontiers allé servir mais je n'y osay aller pourtant qu'il failloit paier comme la coustume est de X pièces d'or l'une et aussy je ne le laissay point tant pour la despensse de la perte de l'or ou argent mais je le fis pourtant que souvent on est espluquiés et ne scait on de quy, il n'est nul besoing que estrangiers sachent quelle choze on porte et sy on a argent ou non. La coustume est fort maulvaise et ossy dangereuse, c'est asses pour mourdrir les gens [...], J.T., f. 277)». 
33. Sur ce type de reliquaires lire Beate FRICKE, «Visages démasqués - un nouveau type de reliquaire chez les Anjou ", dans Dominic OLARIU, Le portrait individuel. Réflexions autour d'une forme de représentation, XIII ${ }^{e}-\mathrm{XV}^{e}$ siècles, Bern, P. Lang, 2009, p. 35-63, sur le reliquaire de Saint-Maximin, p. 44-45. Sur le contexte général de ce sanctuaire bien connu on peut lire Bernard MONTAGNES, « Le pèlerinage provençal à Marie-Madeleine au $\mathrm{xv}^{\mathrm{e}}$ siècle ", Revues des sciences philosophiques et théologiques, n 84, 2001, p. 679-695.

34. Christian SAPIN, "L'autel, son rôle et sa place dans la crypte ", dans Anne BAUD (dir.), Espace ecclésial et liturgie au Moyen-Âge, Lyon, Maison de l'Orient et de la Méditerranée, 2010, p. 331-346. 35. Meredith COHEN, "An Indulgence for the Visitor: The Public at the Sainte-Chapelle", Speculum, 83/4, 2008, p. 840-883. Lire aussi Mary whiteley, « Public and Private Space in Royal and Princely Châteaux in Late Medieval France », dans Annie RENOux (dir.), Palais royaux et princiers au Moyen Âge, Le Mans, Université du Maine, 1996, p. 71-76.

36. La charte réglant les conditions du dépôt à Toulouse du saint Suaire de l'abbaye de Cadouin a été largement étudiée par Michelle FouRNIÉ, «Une municipalité en quête de reliques. Le saint Suaire de Cadouin et son dépôt à Toulouse à la fin du Moyen Âge ", Mémoires de la Société Archéologique du Midi de la France, t. LXXI, 2011, p. 127-162, notamment p. 139. Elle est conservée aux A.M de Toulouse, cote II14/2, je cite ici la copie GG790, f. 13, limitant les dates des ostensions puis précisant: "quam amplius in anno ostendi displicatum et extensum valeat, nisi forsan dominus noster papa, reges, cardinales, duces, comites vel alie eque nobiles ac notabiles valide persone vellent dictum sanctum jocale, causa devociones secrete, videre. " Pour Besançon, on peut s'appuyer sur la synthèse d'Andrea NIColotTI, Le Saint Suaire de Besançon et le chevalier Othon de La Roche, Besançon, Franche-Bourgogne, 2015. Les dates d'ostension sont fixées par les délibérations capitulaires, A.D. du Doubs, G192, 8 août 1523, f. $236 \mathrm{v}^{\circ}$; dès le 11 mai 1524 on mentionne une ostension pour la dame de Beaujeu accomplie «secrete » dans la sacristie de la cathédrale, f. $278 \mathrm{v}^{\circ}$, d'autres le sont dans les décennies suivantes (en $1540, \mathrm{G} 193, \mathrm{f} .588 \mathrm{v}^{\circ} ; 1553$, G195 f. 490 ${ }^{\circ} ; 1558$, G196, f. $160 \mathrm{v}^{\circ} ; 1559$, f. $206 \mathrm{v}^{\circ}$ ). Le 14 juin 1581, il est réaffirmé qu'on ne doit plus montrer le saint Suaire autrement que lors des deux dates coutumières, mais dès le 13 octobre il est dit que les capitulaires dérogeront au statut contraire (" derogaverunt dicto statuto ») et montreront le suaire "complicato et non extenso" (G198, f. $248 \mathrm{r}^{\circ}$ et f. $\left.255 \mathrm{v}^{\circ}\right)$. En 1585 , les chanoines statuent à nouveau : on ne refusera pas de montrer le saint Suaire aux princes et grands nobles venus de loin («aliquos magnates nobiles aut proceres eo longuinquo ad hanc civitatem advenire. ", G199, f. 6r').

37. «Le pape nous fit monstrer le sainct Véronicle et tous les reliquaires dudict Romme comme on a acoustume de les voir en la bonne sepmaine [la Semaine sante] mais ce fut à huis clos, c'est à dire que quand nous les pèlerins estions venus dedens les églises on fermoit les huys. »

38. «Et incontinent que ceulx de la ville perçuprent que les prebstres alloient pour le moins monstrer, incontinent le peuple de ladicte vallée $\mathrm{y}$ vint à sy très grand force que nous ne le poviesmes veoir. [...] ilz prindrent de longues escorries et se s'en vindrent frappans à tors et à travers tant parmy leur visaiges comme sur leurs testes encoires ilz n'y acomptèrent riens et nous fallit este là une très grande espace. Et adonc ce veant ilz se boutèrent jusques audict huis lequel estoit sur la rue et le fermèrent par vive force. Et après ilz s'en vindrent à l'huys de par dedens l'église et se boutèrent ceulx quy estoient en ladicte cappelle à force dehors. »

39. A.D. du Doubs, G193, f. 556, date du 25 novembre 1539.

40. La crainte des vols de reliques, étudiés pour l'époque carolingienne dans le fameux ouvrage de Patrick GEARY, Le vol des reliques au Moyen Âge, Pierre-Emmanuel Dauzat (trad.), Paris, Aubier, 1993 [1 $1^{\text {ère }}$ éd. 1978] est très présente dans notre corpus (à Saint-Maximin par exemple, H.W., p. 507).

41. « Fut amenée sur le char avec ledict corpz sainct [...]. » Or, « quand on ne peult descendre en bas pour saluer ledict sainct, on allume et requiert-on ledict sainct à ceste dicte painture. » 
42. On retrouve ce système à Saint-Denis, Antonio de Beatis décrivant une sculpture en or massif représentant dans l'église haute la tête du saint conservée dans la crypte (A.B., p. 134). Philippe Cordez évoque la question de l'image des reliques dans Philippe CORDEZ, « Reliquien und ihre Bilder: Zur Ablassvermittlung und Bildreproduktion im Spätmittelalter», dans Kristin MAREK, Raphaèle PREISINGER, Marius RIMMELE et Katrin KÄRCHER (dir.), Bild und Körper im Mittelalter, Munich, Fink, 2006, p. 273-286.

43. «Lequel pillier est enclos de fer et y a ung huis pour entrer dedens. Et dict que une personne quy seroit en péchet mortel n'y polroit entrer mais je n'y entray pas [...]».

44. «On œuvre encoires par terre comme une grand sallière dont le couvercle est de layton auquel y a ung crucifix et par ledict trou ou pertuis, lequel est environ aussy grand comme ma paulme, je regarday comme pour cuider voir ledict corpz du glorieux sainct Nicolas, mais on y percoipt riens, synon quand on a de la chandeille, alors on percoipt tout en bas ainssy comme une lampe rendant grand clareté, laquelle est plaine d'huylle. [...] on en donne à cescun pèlerin une ampoullette dont pour ma part je trouvay la manière d'en avoir trois. »

45. Jean-Claude schмiтt, La raison des gestes, Paris, Gallimard, 1990, p. 82-84, applique au geste le concept de Claude LÉVI-STRAUSS, « L'efficacité symbolique », dans Claude LÉVI-STRAUSS, Anthropologie structurale, Paris, Pion, 1958, p. 205-226.

46. C'est la métaphore qu'Alfred Gell appuie sur le Peer Gynt d'Ibsen dans sa réflexion sur « Les idoles concentriques et la personnalité fractale ", Alfred GELL L'art et ses agents, trad. de l'anglais par Sophie Renaut et Olivier Renaut, Dijon, Les Presses du réel, 2009, p. 168-174.

47. La question de l'accès des femmes aux reliques a été posée par Julia Smith pour des périodes antérieures ; Julia SMITH, « Women at the tomb : Access to relic shrines in the early Middle Ages », dans Kathleen mitchell et Ian wood (dir.), The World of Gregory of Tours, Leyde, Brill, 2002, p. 163-180. Elle apparaît dans notre corpus ; voir aussi J.T., f. 41 et 58 des chapelles interdites aux femmes. Au contraire en Flandres, Antonio de Beatis note que femmes prennent soin des autels et ont la garde des reliques (A.B., p. 123).

48. «Nous partismes de Tres [Maastricht] et allaimes couchier à Ayx et trowaimes tant de monde par le chemin que c'estoit chose merveilleuse. Et quant nous vinmes sus la montaigne au dessus de Ayx, nous vimes qu'ils sembloit que toutte l'église fût en feu et en flammes, de fource des lampes qui ardoient entour de la dite église, et il estoit presque nuit, par quoy les dites lampes se moustroient mieulx et puis touttes les grosses cloches sonnoient, qui estoit belle chose à veoir et à oyr de dessus la montaigne. »

49. «Nous avions le regairt sus la plaice d'ung des coustés de la dite église, là où nous voions tant grant puple en la dite plaice que l'on ne veoit que testes et encore autant sus les maixons. »

50. Alfred GELL, op. cit.

51. Thomas GOLSENNE, "L'Ornement est-il animiste?", Histoire de l'art et anthropologie, Paris, coédition INHA / musée du quai Branly, 2009, http://journals.openedition.org/actesbranly/282 (consulté le 7/08/2019).

52. "L'on voit venir biaulcopt de torches et de pillers allumés et puis viengnent biaulcopt de gens d'église, tous revetus en riches habis avec riches croix et yaue benoitte et riches encensiers d'or et d'airgent et tout en belle ordonnance viengnent au luing des aillées devant dites et enmey lieu d'eulx et du luminaire et des encensiers sont deux prélats bien vetus d'or et d'airgent qui pourtent sur leurs espaulles ung rond baton comme d'une lance, doré de fin or, sus lequel baton est mise et posée la précieuse et digne chemize de nostre dame et est ploiée en plusieurs plis sus leur baton et le pourtent comme on porteroit une fierte, et dessus la dite chemize y ait ung biaulx draps de soye et dessus le drapz de soye y ait ung biaulx draps d'or, et voint ainsy tous cheminant jusques à tant qu'ils viengent en belle ordonnance à l'ung des lieux ordonnés, là où le sermon ait esté fait ; et là adonque se arestent et en grande revérence, décowrent la dite chemise du drapz d'or et de celluy de soye et adoncque tout le puple est en genoulx la teste descouverte et les 
mains joinctes [...]. Et adoncque les prélas prengnent la dite chemise qui est ploiée comme dit est et en grande honneur et révérence la laissent ailler de ses plois et l'étendent tout du loing au dehors dites aillées sus ung aultre drapz d'or, à la veue d'ung chacun et adoncque vous diriez que tout le monde tremble du grant bruit des cornets et du cri des hommes et femmes qui crient miséricorde et n'y ait homme que les cheveux ne luy dressent en la teste et que les lairmes ne viengnent à l'euil. »

53. L'hymne " Maria ergo accepit libram unguenti », " Marie, prit une livre d'onguents ", évoque l'onction par Marie-Madeleine des pieds et des cheveux du Christ, d'après Jn, 12, 3.

54. "Quand on le doibt monstrer, on va premiers sonner une clochette et adont viennent premiers les novices et le thésaurier de ladicte église tous revestus à tout torses et chandeilles allumées avec encens fort honnorablement et là chantent cest anthienne Maria ergo ... , aussy la collecte ».

55. Jean-Claude ScHMITT, Le corps des images, Paris, Gallimard, 2002, p. 282-284.

56. Outre l'abondante littérature sur les reliquaires, on pense bien sûr à l'étude de Catherine Vincent sur les luminaires, insistant régulièrement sur leur place auprès des corps saints, Catherine VINCENT, Fiat Lux, Lumière et luminaires dans la vie religieuse en Occident $d u$ XIII siècle au début du XVI ${ }^{e}$ siècle, Paris, Cerf, 2004.

57. Sur les écrits et les images autour des reliques, Paul Bertrand a interrogé les authentiques, c'est-à-dire les étiquettes conservées avec les reliques, Paul BERTRAND, « Authentiques de reliques : authentiques ou reliques?", Le Moyen Age, t. CXII, n², 2006, p. 363-374, en ligne: http:// www.cairn.info/revue-le-moyen-age-2006-2-page-363.htm (consulté le 04/06/2019). Philippe Cordez évoque les livrets de pèlerinage, illustrés et les images de reliques dans Philippe CORDEZ, "Wallfahrt und Medienwettbewerb », op. cit. et Philippe CORDEZ, « Reliquien und ihre Bilder », op. cit. Il évoque aussi l'implication des inventaires dans le dispositif des ostensions, Philippe CORDEZ, Trésor, mémoire, merveilles, op. cit., p. 94.

58. On connaît le cas de l'ostension de la Couronne d'épines des mains de saint Louis, mais aussi du saint Sang de Westminter porté par Henri IV, étudié par Nicholas Vincent (Nicholas VINCENT, The Holy Blood. King Henri III and the Westminster Blood Relic, Cambridge University Press, 2001, fig. 5 et 6); notre corpus comporte des laïcs acteurs des monstrations, mais pas de monstrations de reliques réalisées par des laïcs.

59. On pense par exemple à l'étude classique de Richard C. TREXLER, Public Life in Renaissance Florence, Londres, Cornell University Press, 1991.

60. Pierre-Olivier DITTMAR, « Cachez ce saint », op. cit., p. 97.

61. Parmi les ordres pris par la ville pour la fête du saint Suaire, dans les Délibérations du syndic de 1514 et 1519 (A.D. de Savoie, 189Edépôt80, f. 294rº ) : Item que tot home qui mestier naz et tous bellitres et coquyns doibgent vuyde laz ville et les franchisses dedans XXIIII heures sur laz payne de les forche ".

62. Valeria GENOVESE, Statue vestite e snodate. Un percorso, Pise, Edizioni della Normale, 2011, p. 45-65.

63. Lire Megan HoLmES, The Miraculous Image in Renaissance Florence, New Heaven, Yale University Press, 2013, p. 221.

64. Dans le même manuscrit, on trouve aussi une image de saint Louis montrant la sainte Croix depuis un échafaud, enveloppée dans un linge, f. 141v.

65. Guillaume Durand évoque l'ampoule de l'onction qui après sa bénédiction doit être «en partie couverte, en partie découverte (ex parte cooperta ex parte discooperta) » à l'image du corps du Christ au tombeau, à moitié nu, manifestant ses deux natures divine et humaine; Guillelmi Duranti Rationale divinorum officiorum, Davril ANSELME et Timothy M. THIBODEAU (éd.), CCCM, t. CIX, Turnhout, Brepols, 1995, livre VI, chapitre LXXIV, 18. 
66. Caroline вynum, Christian Materiality. An Essay on Religion in Late Medieval Europe, Cambridge, MIT Press, p. 256-259.

67. Francesca SBARDELLA, «La fabrique des reliques. Manipulations et production de sacré dans la clôture ", Conserveries mémorielles, $\mathrm{n}^{\circ} 14,2013, \S 11$, mis en ligne le 01 juillet 2013, http:// journals.openedition.org/cm/1531 (consulté le 5/06/2019).

68. Sur ce geste voir Jean-Claude sснміт, La raison des gestes, op. cit., p. 346-350, mais encore Gia TOUSSAINT, Kreuz und Knochen, op. cit., p. 15-17.

69. Dans l'ouvrage de Jean-Claude SchmitT, La raison des gestes, op. cit., le troisième chapitre traite du mouvement descendant de la main de Dieu émergeant des cieux.

70. «La clergie par ordre [...] moustre au puple qui est en bais, y celui st chief et le retourne le prélat qui le tient, sens que dessus dessoubz, pour moustrer le tais de la tête toute nus, car il est tout guarni d'airgent ; mais sus la teste il y ait une petite plaitine qui se lièwe, et adont sembloit, que tout deust fendre de fource de courner et businer, tellement que l'on plouroit quasy de joye. »

71. Jacques CHIFFOLEAU, «Les processions parisiennes de 1412 : analyse d'un rituel flamboyant ", Revue historique, t. 284, 1990, p. 37-76, (notamment p. 39-40).

72. Ibid., p. 73.

73. Sur la génuflexion et les autres postures de prières, lire Jean-Claude schмiтT, La raison des gestes, op. cit., p. 295-302.

74. «Le plus espeditivement qui est possible, affin de laisser la devotion en la mémoire des assistans, là où si la chose est prolongée, la devotion se perd, et la confusion y arrive, par le moyen des chapelez que lon jette contre le saint linge pour les faire toucher, et le tumulte qu'il s'en faict ", A.M. de Besançon, ms. 109, F. Guénard, Liber caeremoniarum et officiorum divinorum, p. 144, les archives capitulaires évoquent aussi en 1617 les jets de couronne de fleurs, A.D. du Doubs, G203, cité par Andrea NICOLOTTI, Le Saint Suaire de Besançon, op. cit., p. 17.

75. «Ce qu'il fut faict : et est la chose la plus digne et la plus piteuse que je veis jamais et n'est pas crestien à mon advis, que en la voyant n'a cremeur en soy et plus n'en dis ", Jean GALLOT, « Voyage à Rome par dom Edme, abbé de Clairvaux ", publié par AUGUSTE HARMAND, Mémoires de la Société d'agriculture, des sciences, arts et belles-lettres du département de l'Aube, année 1849/2 (p. 147-235) p. 228.

76. Sur les émotions collectives, on peut lire l'ouvrage de Barbara H. ROSENWEIN, Emotional Communities in the Early Middle Ages, Cornell University Press, 2006 ainsi que le chapitre "L'émotion commune ». Lire aussi Damien BOQUET et Piroska NAGY, « La ferveur religieuse, une émotion collective ", dans Damien BoQUET et Piroska NAGY, Sensible Moyen Âge. Une histoire des émotions dans l'Occident médiéval, Paris, Seuil, 2015, p. 303-346.

77. Denis BRUNA, Enseignes de pèlerinage et enseignes profanes, Paris, Réunion des Musées Nationaux, 1996 et Denis BRUNA, Enseignes de plomb et autres menues chosettes du Moyen Âge, Paris, Éditions du Léopard d'or, 2006.

78. On bénéficie de surcroît d'un outil précieux pour leur étude, la base de données néerlandaise Kunera, en ligne, qui recense nombre de ces objets et que j'utilise pour cette enquête, URL: http://kunera.nl/.

79. Voir les documents collectés par Philippe CORDEZ, «Wallfahrt und Medienwettbewerb», op. cit.

80. Maurice B. MCNAMEE, Vested Angels. Eucharistic Allusions in Early Netherlandish Paintings, Louvain, Peeters, 1998.

81. Thomas воHL, Le décor du cloître de l'abbaye de Cadouin, Mémoire de recherche de l'École du Louvre, 2010, I, p. 31-32. 


\section{RÉSUMÉS}

Plusieurs pèlerins de la fin du Moyen Âge ont écrit des récits de leurs pèlerinages, décrivant leurs visites aux reliques se trouvant sur leur route et la manière dont elles leur étaient montrées. En s'appuyant sur des textes rédigés entre 1460 et 1520, l'article interroge les modulations de ces pratiques de monstration, réalisées publiquement à des dates et des heures fixes ou occasionnellement, à la requête des pèlerins. Les reliques montrées y apparaissent comme des corps-images dont les institutions organisent avec soin la visibilité. Mais plutôt que de s'attarder sur l'apparence des reliques, les pèlerins sont surtout attentifs à la manière dont elles sont montrées, par qui et dans quels lieux, avec quels ornements, occasionnant quelle réaction chez les fidèles et chez eux-mêmes. De la sorte la relique organise autour d'elle un dispositif qui inclut des objets, des corps agissants, le corps social, s'exposant avec elle.

Many pilgrims from the late Middle Ages recounted their pilgrimage, describing their visits to the relics along their route and how they were shown to them. Based on texts written between 1460 and 1520, this paper analyses the different ways in which this monstration was taking place, publicly, at fixed times, or occasionally, on the pilgrims' query. The displayed relics appear as body-images, whose visibility is organized by the institutions. However, rather than their appearance, pilgrims describe principally the way the relics were displayed, by whom and where, with which kind of adornment, the impression they created to the crowd and to themselves. Therefore, a display is organized around the relic, including objects, acting bodies, and society that is displayed with it.

\section{INDEX}

Mots-clés : christianisme, reliques, images saintes, reliquaire, pèlerinage

Keywords : Christianism, relics, holy images, shrine, pilgrimage

\section{AUTEUR}

\section{NICOLAS SARZEAUD}

Nicolas Sarzeaud est doctorant en histoire de l'art médiéval à l'EHESS sous la direction d'Étienne Anheim et Pierre-Olivier Dittmar. Il étudie la série des saints Suaires imprimés à la fin du Moyen Âge et il s'intéresse à l'esthétique de l'empreinte, à l'économie de la démultiplication de l'image sainte, ainsi qu'aux implications politiques et sociales de tels cultes. Il travaille par ailleurs sur l'usage des fac-simile dans le patrimoine contemporain. 